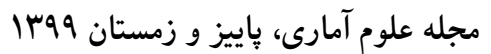

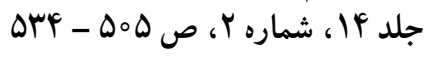 \\ DOI: $10.29252 /$ jss.14.2.505
}

برآورد بيزى پارامتر تنش-مقاومت تحت نمونههاى سانسور فزاينده پيوندى در توزيع

$$
\begin{aligned}
& \text { اكرم كهنسال'، نفيسه آل محمد'، فاطمه عزيززاده؟ } \\
& \text { اكروه آمار، دانشگاه بين المللى امام خمينى (ره) }
\end{aligned}
$$

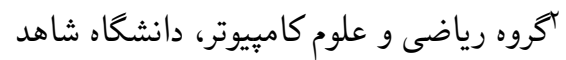

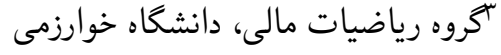

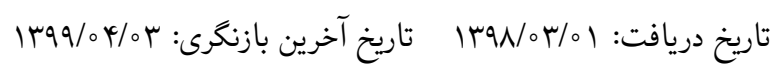

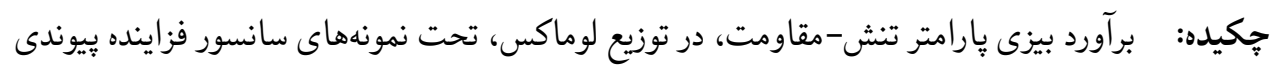

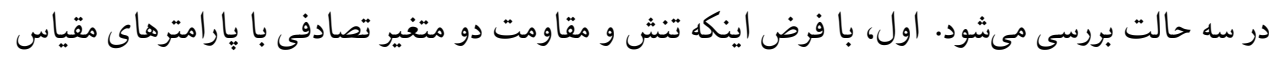

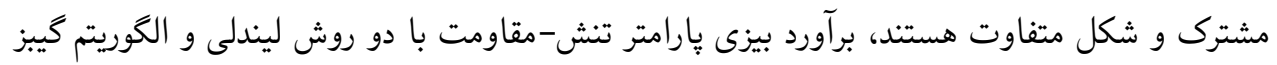

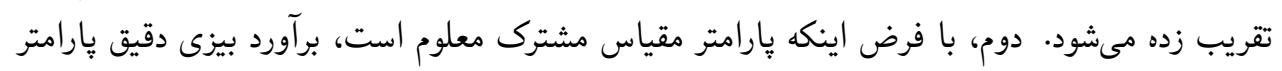

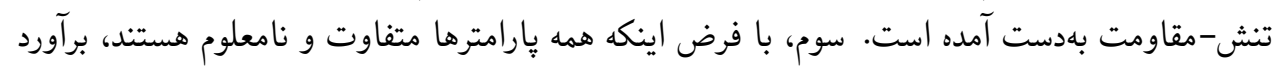

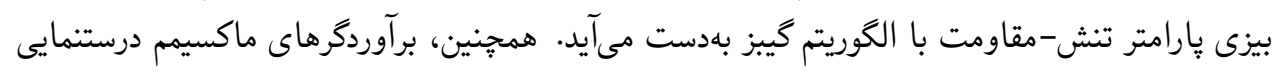

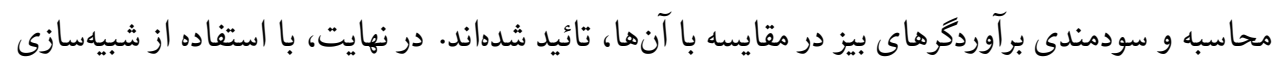
مونت كارلو، روشهاى مختلف ارزيابى شده و يك مجموعه داده واقعى تحليل مى بـودود. وازدهاى كليدى: يارامتر تنش-مقاومت، تقريب ليندلى، توزيع لوماكس، سانسور فزاينده بيوندى 
يكى از مسائل در قابليت اعتماد، استنباط آمارى براى بارامتر تنش-مقاومت، (بن

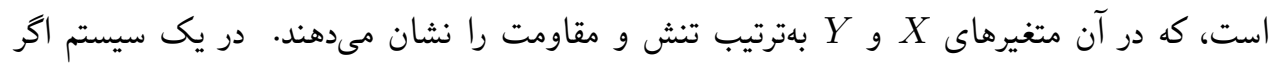

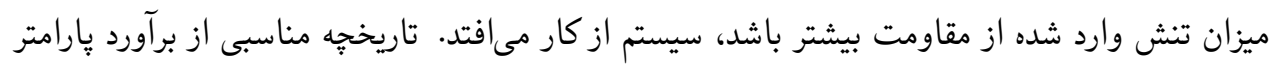

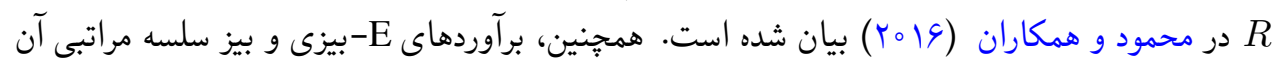

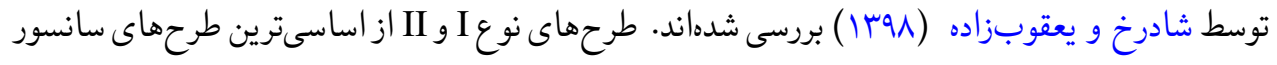

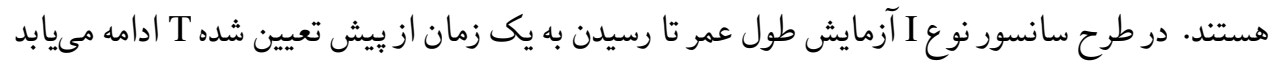

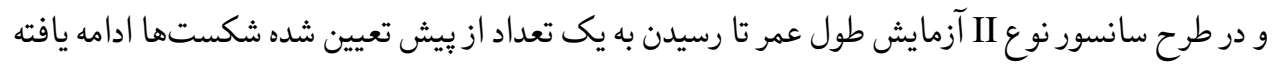

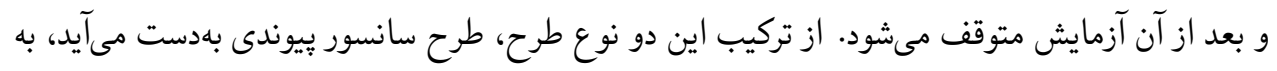
طورى كه آزمايش طول عمر در زمان

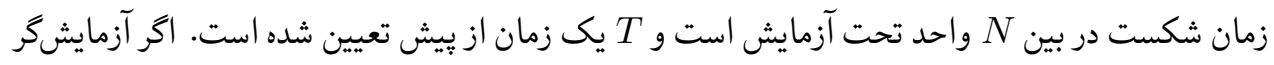

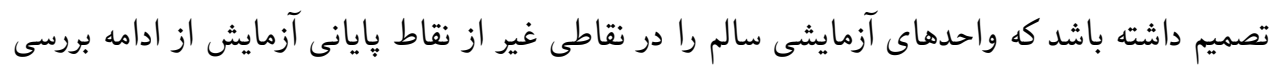

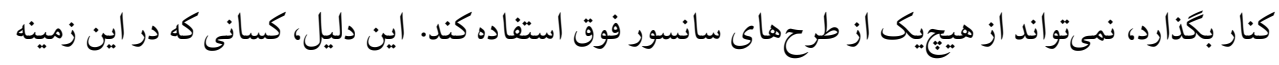

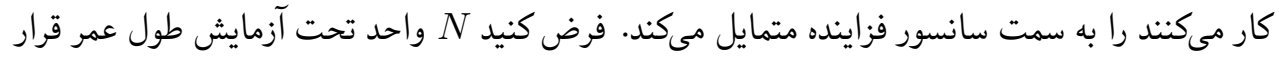

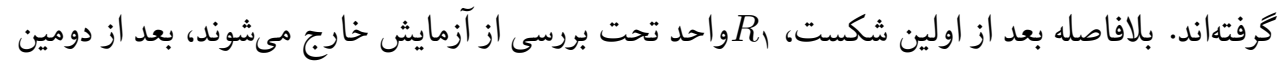
شكست، إين اين حالت طرح سانسور با $\left(R^{\prime}\right.$ انس

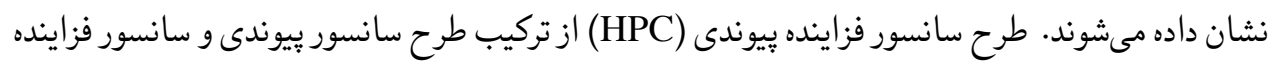

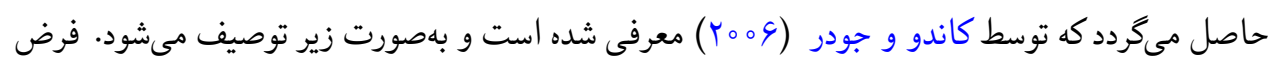

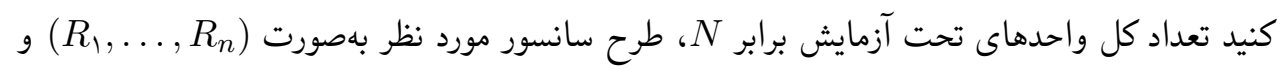
فزايندة و

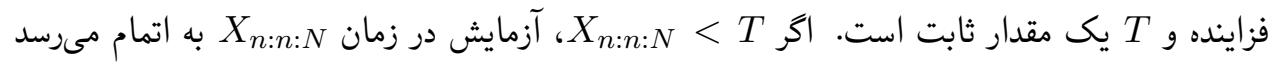
و نمونه در دسترس بهصورت

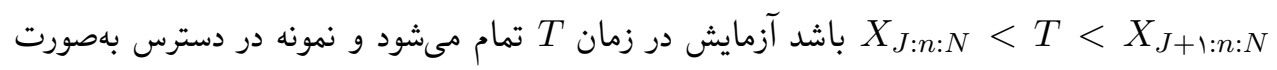

$$
\text { 落: } X_{1: n: N}<\cdots<X_{J: n: N}
$$



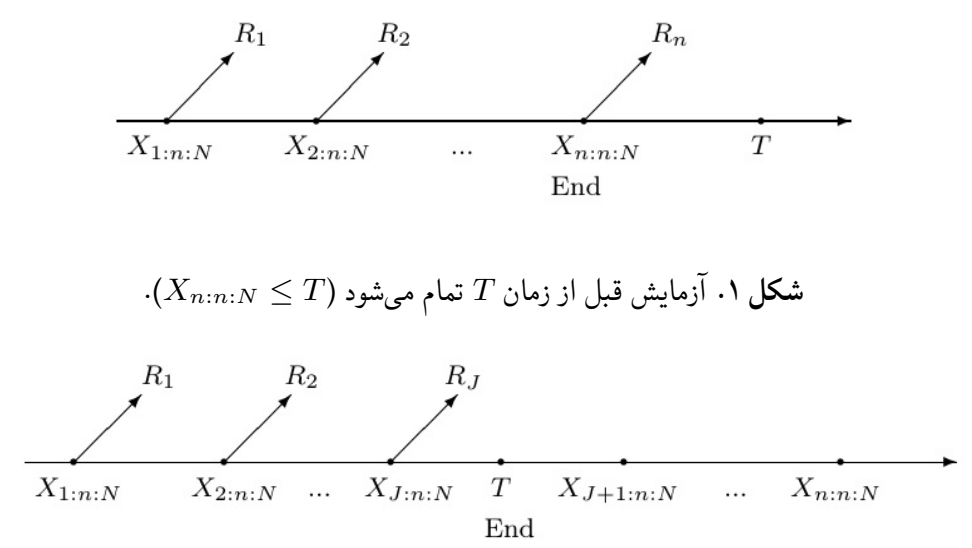

$$
\text { شكل r. آزمايش در زمان T تمام مىشود }\left(X_{J: n: N} \leq T \leq X_{J+1: n: N}\right.
$$

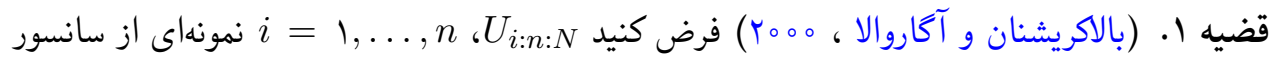

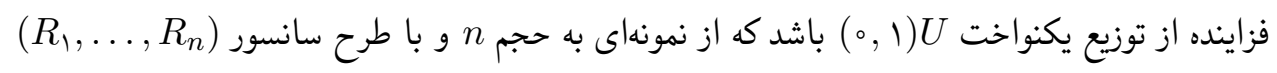

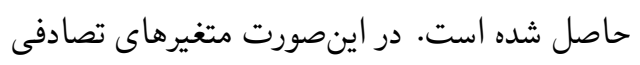
$V_{\uparrow}=\frac{1-U_{n: n: N}}{1-U_{n-1: n: N}}, \quad V_{\uparrow}=\frac{1-U_{n-1: n: N}}{1-U_{n-\uparrow: n: N}}, \ldots, V_{n}=1-U_{1: n: N}$

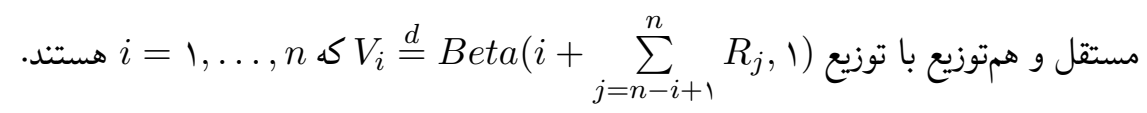
الكوريتم ا. توليد آمارههاى ترتيبى سانسور فزاينده:

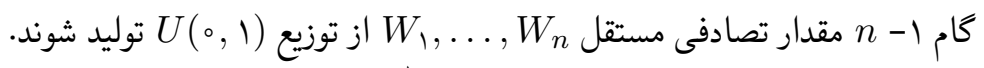
كام ץ- به ازاى

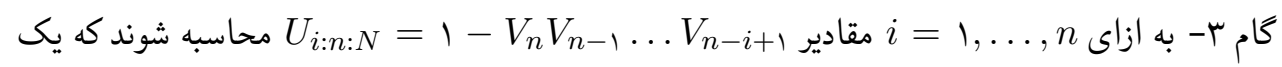

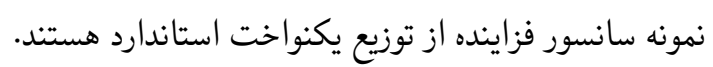

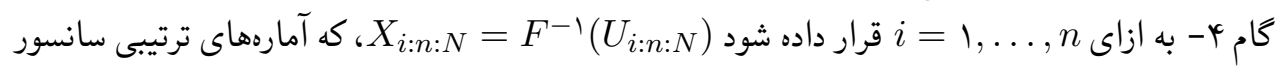
فزاينده از توزيع دلخواه (·) فرع ا. روش توليد دادههاى سانسور فزاينده بِيوندى به اينصورت است كه ابتدا براى n، N و T و طرح 
برآورد بيزى ثارامتر R در توزيع لوماكس

سانسور $\left(X_{1: n: N}, \ldots, X_{n: n: N}\right.$ توليد مىشود. اخر $>$ >

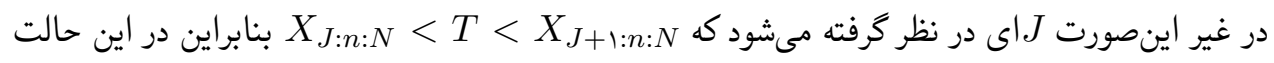
نمونه با بنابر بالكريشنان و آكاروالا (Yoo0)، تابع درستمايى نمونهاى HPC بهصورت

$$
L(\theta, \beta) \propto \prod_{i=1}^{J} f\left(x_{i}\right)\left[1-F\left(x_{i}\right)\right]^{R_{i}}[1-F(T)]^{R_{J}^{*}}
$$

است، كه در آن

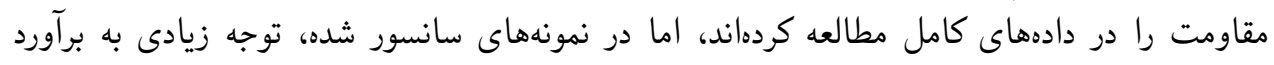

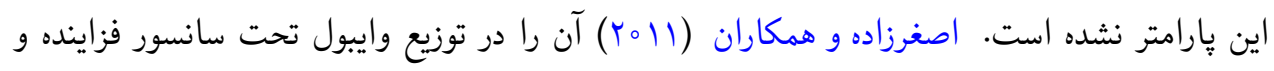

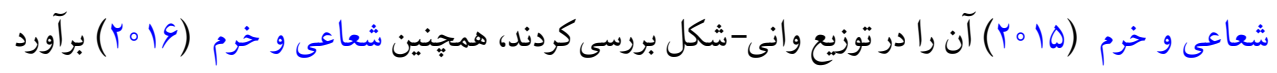

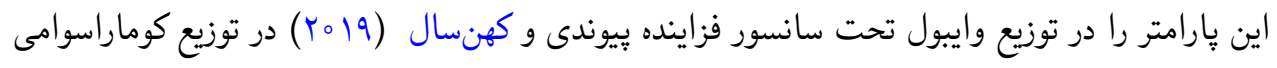

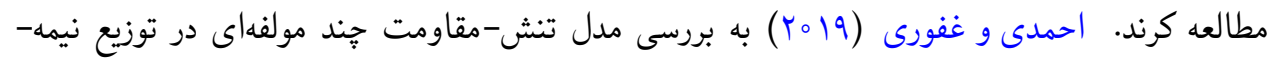

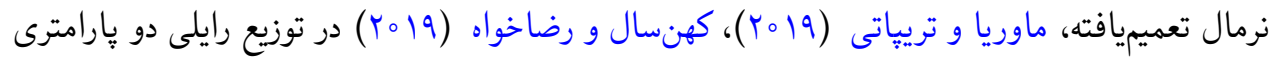

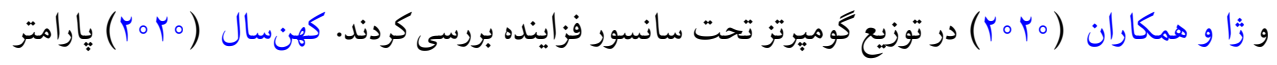

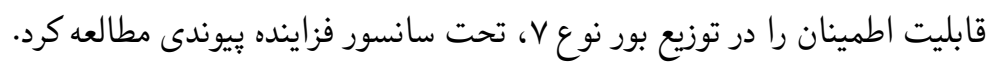

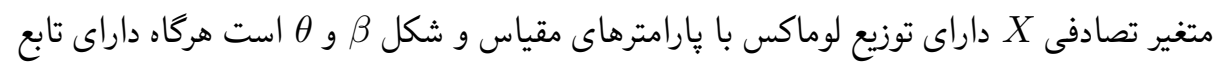

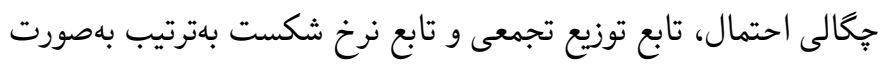

$$
\begin{aligned}
& f(x)=\beta \theta(1+\beta x)^{-\theta-1}, x>0, \\
& F(x)=1-(1+\beta x)^{-\theta}, x>0, \\
& h(x)=\beta \theta(1+\beta x)^{-1}, \quad x>0 .
\end{aligned}
$$

باشد، كه با نماد

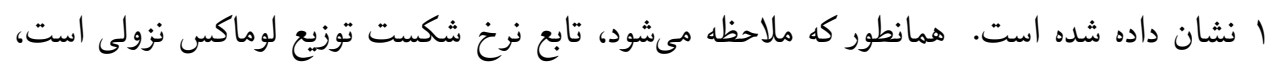


بنابراين اخر در يك مجموعه داده، با بررسىهاى تجربى، نتيجه گرفته شود كه تابع نرخ شكست نزولى است،

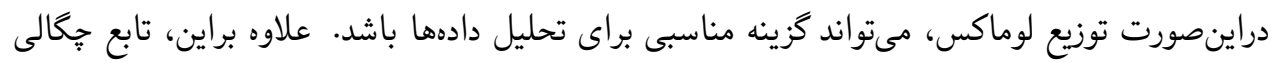

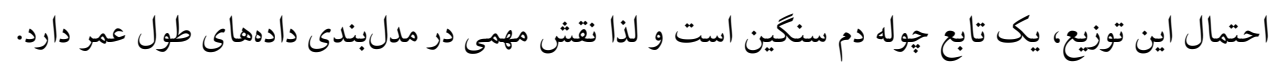

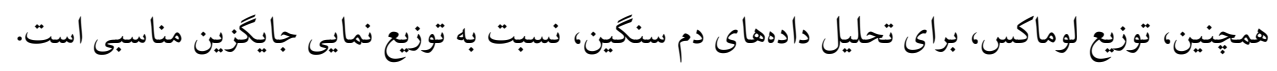

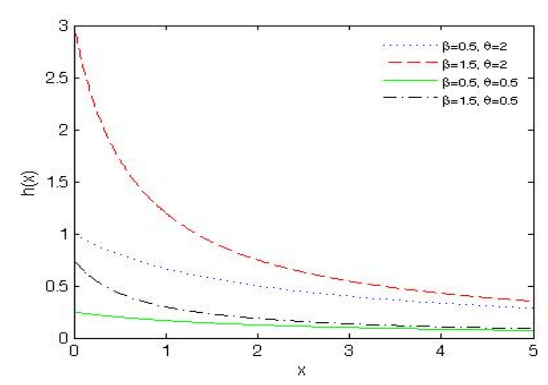

(ب)

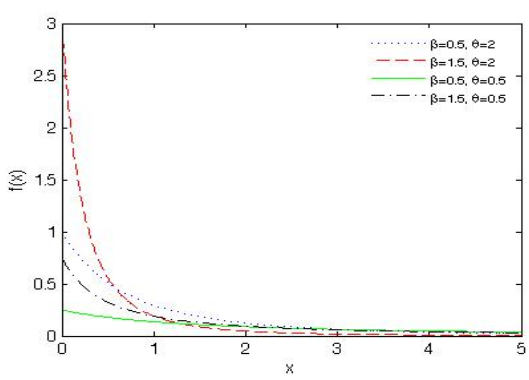

(الف)

شكل r. الف- نمودار تابع جكالى احتمال توزيع لوماكس، ب- نمودار تابع نرخ شكست توزيع لوماكس

هدف اين مقاله برآورد پارامتر تنش-مقاومت تحت سانسور فزاينده يِيوندى در توزيع لوماكس است.

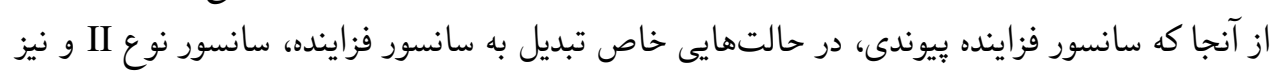

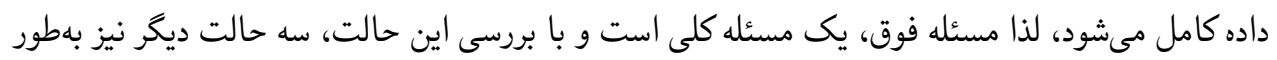

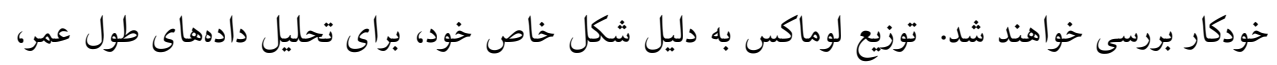
جايكزين مناسبى نسبت به توزيع نمايى و بسيارى از توزيعهاى مهم در نظريه قابليت اعتماد است.

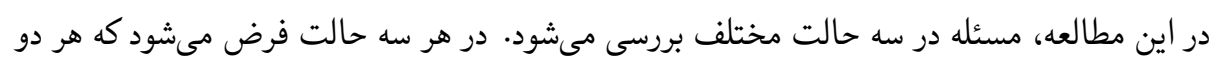

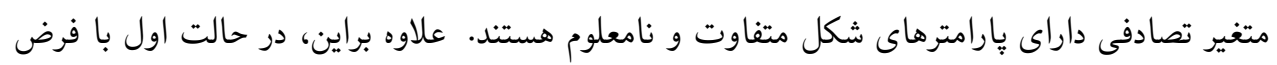

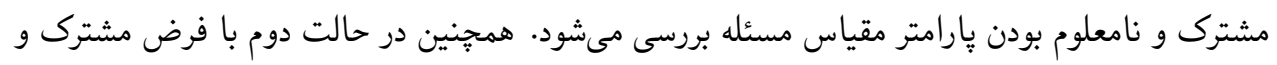

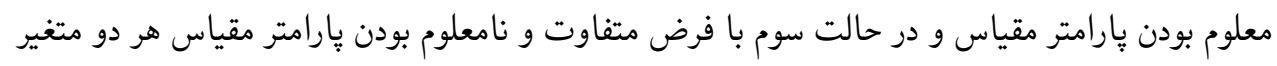

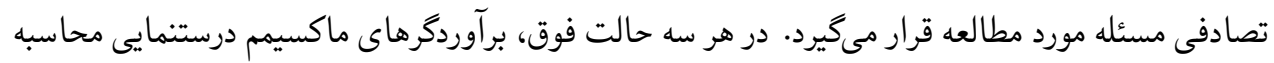

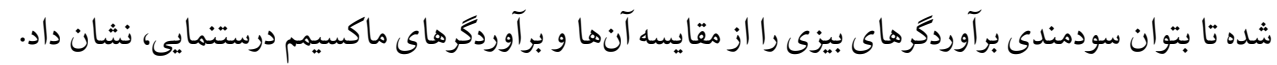

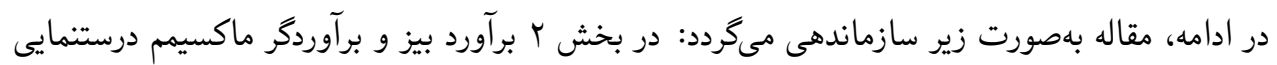

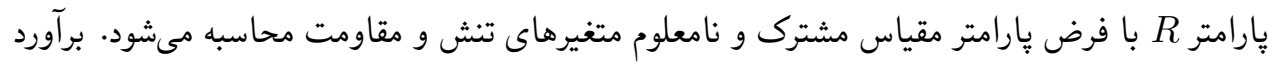

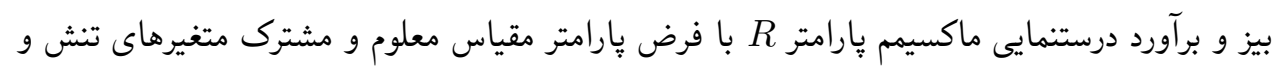


ب... - برآورد بيزى يارامتر R در توزيع لوماكس

$01 \circ$

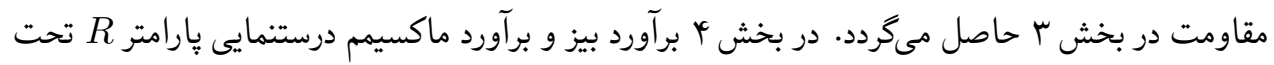

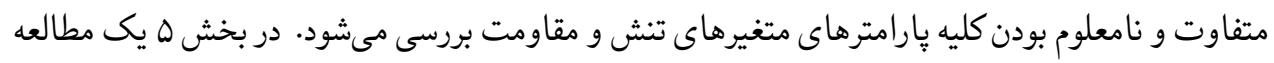

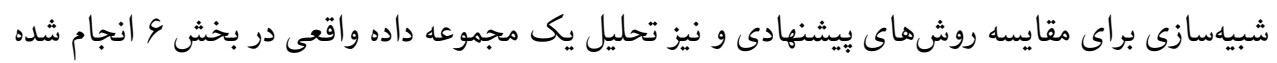
و در نهايت جمعبندى از اين مقاله ارائه مئردد.

\section{r استنباط يارامتر R با يارامتر مشترك و نامعلوم}

$R=\frac{\theta}{\alpha+\theta}$ اكر است. بنابراين، اكر

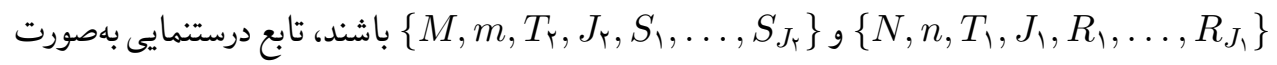

$$
\begin{aligned}
L(\theta, \alpha, \beta) & \propto\left(\prod_{i=1}^{J_{\curlywedge}} f\left(x_{i}\right)\left[1-F\left(x_{i}\right)\right]^{R_{i}}\left[1-F\left(T_{\uparrow}\right)\right]^{R_{J}^{*}}\right) \\
& \times\left(\prod_{j=1}^{J_{\uparrow}} f\left(y_{j}\right)\left[1-F\left(y_{j}\right)\right]^{S_{j}}\left[1-F\left(T_{\uparrow}\right)\right]^{S_{J_{\digamma}}^{*}}\right) .
\end{aligned}
$$

نوشته مىشود. بنابراين تابع درستنمايى برمبناى دادهاى مشاهده شده به صورت

$$
\begin{aligned}
& L(\text { data } \mid \theta, \alpha, \beta) \propto \theta^{J_{\curlywedge}} \beta^{J_{\curlywedge}}\left(\prod_{i=1}^{J_{1}}\left(1+\beta x_{i}\right)^{-\theta\left(R_{i}+1\right)-1}\right)\left(1+\beta T_{1}\right)^{-\theta R_{J_{1}}^{*}} \\
& \times \quad \alpha^{J_{\curlyvee}} \beta^{J_{\curlyvee}}\left(\prod_{j=1}^{J_{\curlyvee}}\left(1+\beta y_{j}\right)^{-\theta\left(S_{j}+1\right)-1}\right)\left(1+\beta T_{\uparrow}\right)^{-\theta S_{J_{\curlyvee}}^{*}} \\
& \propto\left(\prod_{i=1}^{J_{1}}\left(1+\beta x_{i}\right)^{-1}\right) e^{-\theta\left(\sum_{i=1}^{J_{1}}\left(R_{i}+1\right) \log \left(1+\beta x_{i}\right)+R_{J_{1}}^{*} \log \left(1+\beta T_{1}\right)\right)} \\
& \times\left(\prod_{j=1}^{J_{\curlyvee}}\left(1+\beta y_{j}\right)^{-1}\right) e^{-\theta\left(\sum_{j=1}^{J_{\digamma}}\left(S_{j}+1\right) \log \left(1+\beta y_{j}\right)+S_{J_{\digamma}}^{*} \log \left(1+\beta T_{\Upsilon}\right)\right)} \\
& \times \quad \theta^{J_{\curlywedge}} \alpha^{J_{\curlyvee}} \beta^{J_{1}+J_{\curlyvee}} .
\end{aligned}
$$




$$
\begin{aligned}
& \widehat{\theta}(\beta)=J_{1}\left\{\sum_{i=1}^{J_{1}}\left(R_{i}+1\right) \log \left(1+\beta x_{i}\right)+R_{J_{\curlywedge}}^{*} \log \left(1+\beta T_{\uparrow}\right)\right\}^{-1}, \\
& \widehat{\alpha}(\beta)=J_{\curlyvee}\left\{\sum_{j=1}^{J_{\digamma}}\left(S_{j}+1\right) \log \left(1+\beta y_{j}\right)+S_{J_{\digamma}}^{*} \log \left(1+\beta T_{\Upsilon}\right)\right\}^{-1},
\end{aligned}
$$$$
\text { هستند. همجنِين } \widehat{~ ا ز ~ ح ل ~ م ع ا د ل ه ~ غ ي ر ~ خ ط ى ~}
$$

$$
\begin{aligned}
& \frac{J_{1}+J_{\curlyvee}}{\beta}-\sum_{i=1}^{J_{1}}\left(\theta\left(R_{i}+1\right)+1\right) \frac{x_{i}}{1+\beta x_{i}}-\theta R_{J_{1}}^{*} \frac{T_{1}}{1+\beta T_{\uparrow}} \\
& -\sum_{j=1}^{J_{\digamma}}\left(\alpha\left(S_{j}+1\right)+1\right) \frac{y_{j}}{1+\beta y_{j}}-\alpha S_{J_{\digamma}}^{*} \frac{T_{\Upsilon}}{1+\beta T_{\Upsilon}}=。 .
\end{aligned}
$$

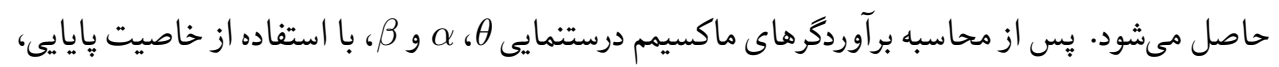
برآوردكر ماكسيمم درستنمايى R برابر بـا برآير

$$
\widehat{R}=\frac{\widehat{\theta}}{\widehat{\alpha}+\widehat{\theta}}
$$

است. اكنون استباط بيزى پارامتر R تحت تابع زيان توانهاى دوم خطا، براى توابع جكالى ييشين

$$
\begin{aligned}
& \pi_{\uparrow}(\theta) \propto \theta^{a_{\uparrow}-1} e^{-b_{1} \theta}, \quad \theta>\circ, a_{\uparrow}, b_{\uparrow}>\circ, \\
& \pi_{\Upsilon}(\alpha) \propto \alpha^{a_{\Upsilon}-1} e^{-b_{\Upsilon} \alpha}, \quad \alpha>\circ, a_{\Upsilon}, b_{\Upsilon}>\circ, \\
& \pi_{\Gamma}(\beta) \propto \beta^{a_{\Gamma-1}} e^{-b_{\Gamma} \beta}, \quad \beta>\circ, a_{\Gamma}, b_{\Gamma}>0 .
\end{aligned}
$$

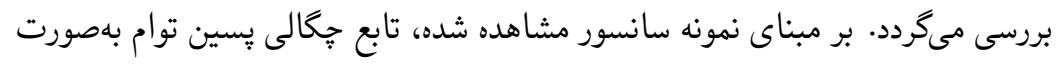

$$
\begin{aligned}
& \pi(\theta, \alpha, \beta \mid \text { data }) \quad \propto \quad L(\text { data } \mid \theta, \alpha, \beta) \pi_{\curlywedge}(\theta) \pi_{\digamma}(\alpha) \pi_{\digamma}(\beta),
\end{aligned}
$$




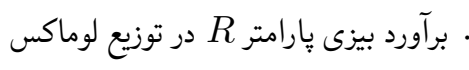

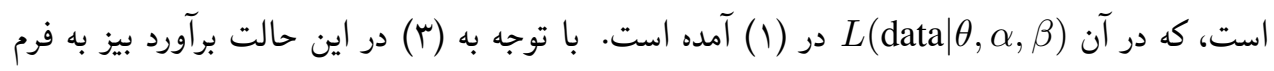

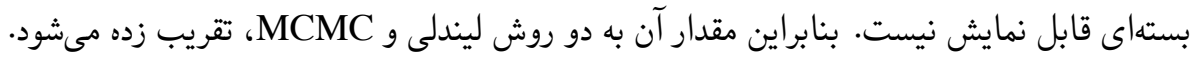

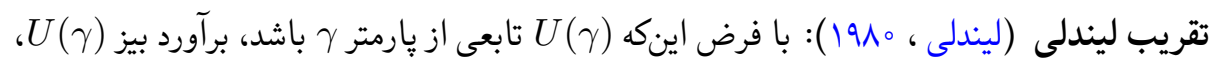

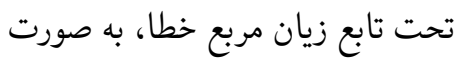

$$
\mathrm{E}(u(\gamma) \mid \text { data })=\frac{\int u(\gamma) e^{\ell(\gamma) \rho(\gamma)} d \gamma}{\int e^{\ell(\gamma) \rho(\gamma)} d \gamma}
$$

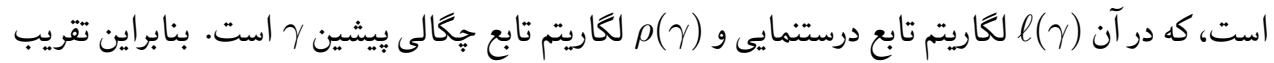

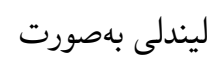

$$
\begin{aligned}
\mathrm{E}(u(\gamma) \mid \text { data }) & =u+\frac{1}{r} \sum_{i} \sum_{j}\left(u_{i j}+r u_{i} \rho_{j}\right) \sigma_{i j} \\
& +\left.\frac{1}{r} \sum_{i} \sum_{j} \sum_{k} \sum_{p} \ell_{i j k} \sigma_{i j} \sigma_{k p} u_{p}\right|_{\gamma=\hat{\gamma}},
\end{aligned}
$$

است، كه در آن ، $\sigma_{i j}$ ،

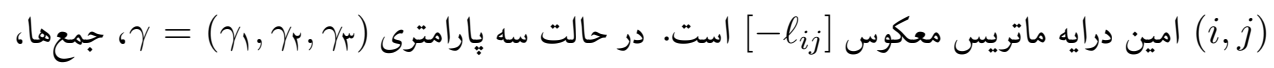
در رابطه (†) بهصورت سادمتر

$$
\begin{aligned}
& \mathrm{E}(u(\gamma) \mid \text { data })=u+u_{1} d_{\uparrow}+u_{\digamma} d_{\digamma}+u_{\digamma} d_{\digamma}+d_{\digamma}+d_{\Delta}
\end{aligned}
$$

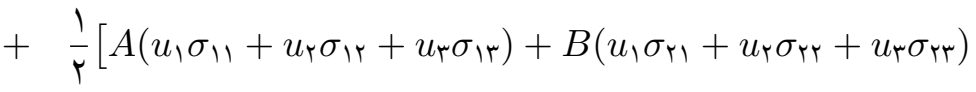

$$
\begin{aligned}
& \left.+C\left(u_{1} \sigma_{r}+u_{r} \sigma_{r r}+u_{r} \sigma_{r r}\right)\right] \text {, }
\end{aligned}
$$

نتيجه مىشوند. در حالت سه بارامترى بايد (ه) را بوسيله برآورد ماكسيم درستنمايى بلبدست آورد، كه در آن،

$$
\begin{aligned}
& d_{i}=\rho_{\uparrow} \sigma_{i \uparrow}+\rho_{\curlyvee} \sigma_{i \curlyvee}+\rho_{\curlyvee} \sigma_{i ץ}, \quad i=1, \Upsilon, \digamma, \\
& d_{\uparrow}=u_{\uparrow \uparrow} \sigma_{\uparrow \uparrow}+u_{\uparrow \uparrow} \sigma_{\uparrow \uparrow}+u_{\uparrow \uparrow} \sigma_{\uparrow \uparrow},
\end{aligned}
$$




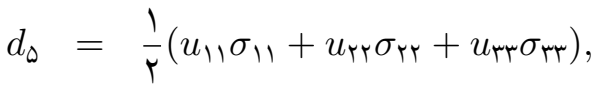

$$
\begin{aligned}
& A=\ell_{111} \sigma_{11}+r \ell_{|r|} \sigma_{\mid r}+r \ell_{|r|} \sigma_{\mid r}+r \ell_{r \mu} \sigma_{r r}+\ell_{r r \mid} \sigma_{r r}+\ell_{r \mu \mid} \sigma_{r r},
\end{aligned}
$$

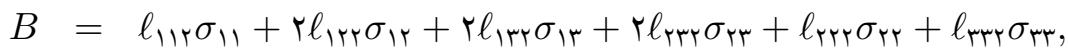

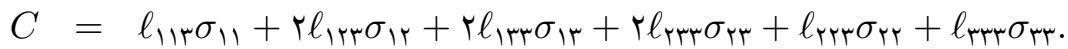

حال با قرار دادن $u=R=\frac{\theta}{\alpha+\theta}\left(\gamma_{1}, \gamma_{\Gamma}, \gamma_{\Gamma}\right)=(\theta, \alpha, \beta)$ مىتوان روابط

$$
\begin{aligned}
& \rho_{\uparrow}=\frac{a_{\uparrow}-1}{\theta}-b_{\uparrow}, \rho_{\Gamma}=\frac{a_{\Gamma}-1}{\alpha}-b_{\Gamma}, \rho_{\Gamma}=\frac{a_{\Gamma}-1}{\beta}-b_{r}, \\
& \ell_{11}=-\frac{J_{1}}{\theta^{r}}, \quad \ell_{r}=-\frac{J_{Y}}{\alpha^{r}}, \quad \ell_{T r}=\ell_{\Gamma}=0 \text {, } \\
& \ell_{1 \Gamma}=-\sum_{i=1}^{J_{1}}\left(R_{i}+1\right) \frac{x_{i}}{1+\beta x_{i}}-R_{J_{1}}^{*} \frac{T_{1}}{1+\beta T_{1}}, \\
& \ell_{\Upsilon \Gamma}=-\sum_{j=1}^{J_{\Upsilon}}\left(S_{j}+1\right) \frac{y_{j}}{1+\beta y_{j}}-R_{J_{\uparrow}}^{*} \frac{T_{\Upsilon}}{1+\beta T_{\Upsilon}}, \\
& \ell_{\Gamma \Gamma}=-\frac{J_{1}+J_{\Upsilon}}{\beta^{\Upsilon}}+\theta \sum_{i=1}^{J_{1}}\left(R_{i}+1\right)\left(\frac{x_{i}}{1+\beta x_{i}}\right)^{r}+\theta R_{J_{1}}^{*}\left(\frac{T_{1}}{1+\beta T_{1}}\right)^{r} \\
& +\alpha \sum_{j=1}^{J_{\curlyvee}}\left(S_{j}+1\right)\left(\frac{y_{j}}{1+\beta y_{j}}\right)^{\Upsilon}+\alpha R_{J_{\digamma}}^{*}\left(\frac{T_{\Upsilon}}{1+\beta T_{\Upsilon}}\right)^{\Upsilon} \\
& +\sum_{i=1}^{J_{1}}\left(\frac{x_{i}}{1+\beta x_{i}}\right)^{r}+\sum_{j=1}^{J_{\curlyvee}}\left(\frac{y_{j}}{1+\beta y_{j}}\right)^{r}
\end{aligned}
$$


برآورد بيزى پارامتر R در توزيع لوماكس

را بهدست آورد، كه در آن

$$
\begin{aligned}
& \ell_{111}=\frac{r J_{1}}{\theta^{r}}, \quad \ell_{r Y r}=\frac{r J_{r}}{\alpha^{r}}, \\
& \ell_{1 \Gamma r}=\sum_{i=1}^{J_{1}}\left(R_{i}+1\right)\left(\frac{x_{i}}{1+\beta x_{i}}\right)^{r}+R_{J_{1}}^{*}\left(\frac{T_{1}}{1+\beta T_{\uparrow}}\right)^{r} \text {, } \\
& \ell_{\Upsilon \Upsilon \Gamma}=\sum_{j=1}^{J_{\Upsilon}}\left(S_{j}+1\right)\left(\frac{y_{j}}{1+\beta y_{j}}\right)^{\Upsilon}+R_{J_{\Upsilon}}^{*}\left(\frac{T_{\Upsilon}}{1+\beta T_{\Upsilon}}\right)^{\Upsilon} \text {, } \\
& \ell_{r \Gamma r}=\frac{r\left(J_{1}+J_{Y}\right)}{\beta^{r}}-r \theta \sum_{i=1}^{J_{1}}\left(R_{i}+1\right)\left(\frac{x_{i}}{1+\beta x_{i}}\right)^{r}-r \theta R_{J_{1}}^{*}\left(\frac{T_{1}}{1+\beta T_{1}}\right)^{r} \\
& -r \alpha \sum_{j=1}^{J_{\digamma}}\left(S_{j}+1\right)\left(\frac{y_{j}}{1+\beta y_{j}}\right)^{r}-r \alpha R_{J_{r}}^{*}\left(\frac{T_{\Upsilon}}{1+\beta T_{\curlyvee}}\right)^{r} \\
& -r \sum_{i=1}^{J_{\uparrow}}\left(\frac{x_{i}}{1+\beta x_{i}}\right)^{r}-r \sum_{j=1}^{J_{r}}\left(\frac{y_{j}}{1+\beta y_{j}}\right)^{r} \text {, }
\end{aligned}
$$

و نيز ساير = =

$$
\begin{aligned}
& d_{i}=\rho_{\uparrow} \sigma_{i \uparrow}+\rho_{\curlyvee} \sigma_{i \curlyvee}+\rho_{\curlyvee} \sigma_{i \curlyvee}, i=1, \curlyvee, \uparrow,
\end{aligned}
$$

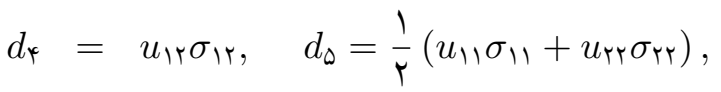

$$
\begin{aligned}
& A=\ell_{11} \sigma_{11}+\ell_{r Y} \sigma_{r \mu}, \quad B=\ell_{Y Y Y} \sigma_{Y r}+\ell_{r \mu r} \sigma_{r Y},
\end{aligned}
$$

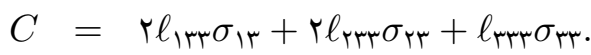

$$
\begin{aligned}
& u_{\uparrow}=\frac{\alpha}{(\alpha+\theta)^{r}}, \quad u_{\Upsilon}=\frac{-\theta}{(\alpha+\theta)^{r}}, \quad u_{\Gamma}=\circ \text {, } \\
& u_{\uparrow}=\frac{-r \alpha}{(\alpha+\theta)^{r}}, \quad u_{\Upsilon \Upsilon}=\frac{r \theta}{(\alpha+\theta)^{r}}, \quad u_{\uparrow \Gamma}=\frac{\theta-\alpha}{(\alpha+\theta)^{r}}, \\
& u_{i r}=\circ, \quad i=1, r, r \text {. }
\end{aligned}
$$




$$
\begin{aligned}
& \text { در نتيجه، تحت تابع زيان توانهاى دوم خطا، برآورد بيز R ببه صورت } \\
& \widehat{R}^{L i n}=u+u_{\uparrow} d_{\uparrow}+u_{\uparrow} d_{\uparrow}+d_{\uparrow}+d_{\Delta}+\frac{1}{\uparrow}\left[A\left(u_{\uparrow} \sigma_{11}+u_{\uparrow} \sigma_{\mid \uparrow}\right)\right.
\end{aligned}
$$

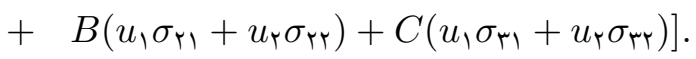

است. توجه شود كه همه بارامترها در مقادير برآورد ماكسيمم درستنمايى $(\widehat{\theta}, \widehat{\alpha}, \widehat{\beta})$ محاسبه مىشوند.

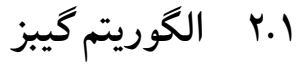

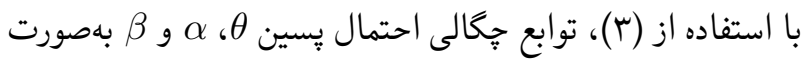

$$
\begin{aligned}
\theta \mid \beta, \text { data } & \sim \Gamma\left(J_{\uparrow}+a_{\uparrow}, b_{\uparrow}+V(\beta)\right), \\
\alpha \mid \beta, \text { data } & \sim \Gamma\left(J_{\Upsilon}+a_{\Upsilon}, b_{\Upsilon}+U(\beta)\right),
\end{aligned}
$$

$\pi(\beta \mid \theta, \alpha$, data $) \propto \beta^{J_{\uparrow}+J_{\digamma}+a_{r}-1} e^{-\theta V(\beta)-\alpha U(\beta)-b_{\digamma} \beta}\left(\prod_{i=1}^{J_{\uparrow}} \frac{1}{1+\beta x_{i}}\right)\left(\prod_{j=1}^{J_{\curlyvee}} \frac{1}{1+\beta y_{j}}\right)$,

$$
\text { بهدست مى آيد، كه در آن }
$$

$$
\begin{aligned}
& V(\beta)=\sum_{i=1}^{J_{\uparrow}}\left(R_{i}+1\right) \log \left(1+\beta x_{i}\right)+R_{J_{\uparrow}}^{*} \log \left(1+\beta T_{\uparrow}\right), \\
& U(\beta)=\sum_{i=1}^{J_{\curlyvee}}\left(S_{j}+1\right) \log \left(1+\beta y_{j}\right)+S_{J_{\uparrow}}^{*} \log \left(1+\beta T_{\uparrow}\right) .
\end{aligned}
$$

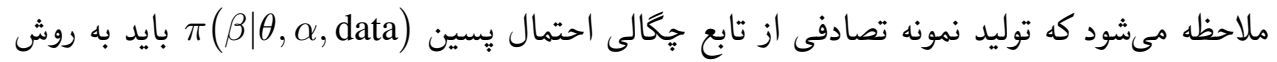
مترويوليس-هستينكز انجام شود. الكوريتم r. برآورد كيبز R: كام ا- با مقادير اوليه $)$

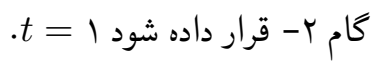
كام ץ- با روش مترويوليس-هستينكز 


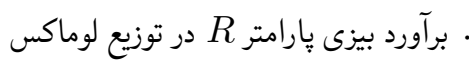

$$
\begin{aligned}
& \text { ( } N\left(\beta_{(t-1)}, 1\right)
\end{aligned}
$$

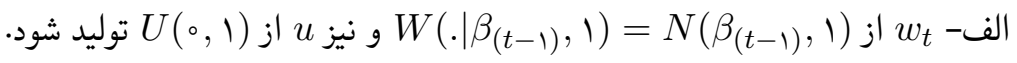

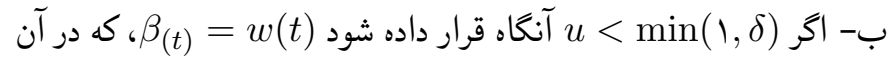

$$
\begin{aligned}
& \delta=\frac{\pi(w(t) \mid \text { data }) W\left(\beta_{(t-1)} \mid w(t), 1\right)}{\pi\left(\beta_{t-1} \mid \text { data }\right) W\left(w(t) \mid \beta_{(t-1)}, 1\right)},
\end{aligned}
$$

$$
\begin{aligned}
& \text { در غير اينصورت به گام الف بازگردد. }
\end{aligned}
$$

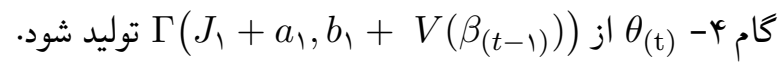

$$
\begin{aligned}
& \text { كام ه- } \\
& \text { كام צ- مقدار }
\end{aligned}
$$

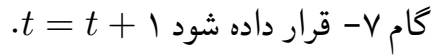

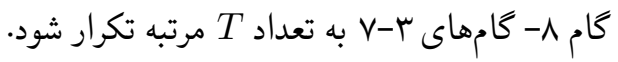

$$
\begin{aligned}
& \text { برآورد بيزى يارامتر R تحت تابع زيان توانهاى دوم خطا به صورت } \\
& \hat{R}^{M C}=\frac{1}{T} \sum_{t=1}^{T} R_{t} .
\end{aligned}
$$

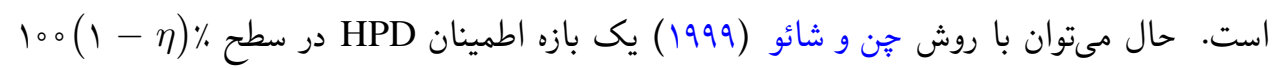
براى بارامتر R ساخت. براى اينكار مقادير تمام بازهاى اطمينان \%

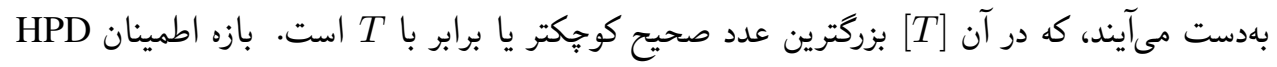

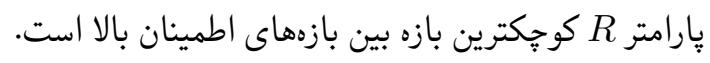

\section{ب استنباط R مبا يارامتر مشترك و معلوم}

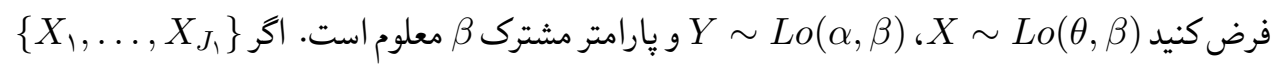

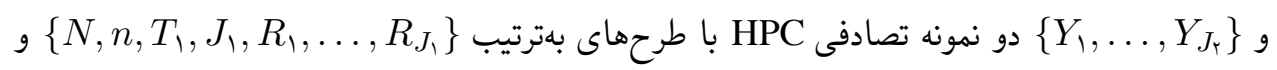
ب باشند، وقتى يارامتر مشترك $\beta$ معلوم باشد، برآورد ماكسيم درستمايى 


$$
\widehat{R}=\left\{1+\frac{J_{\Upsilon}}{J_{1}} \frac{\sum_{i=1}^{J_{1}}\left(R_{i}+1\right) \log \left(1+\beta x_{i}\right)+R_{J_{1}}^{*} \log \left(1+\beta T_{1}\right)}{\sum_{j=1}^{J_{\Upsilon}}\left(S_{j}+1\right) \log \left(1+\beta y_{j}\right)+S_{J_{\Upsilon}}^{*} \log \left(1+\beta T_{\Upsilon}\right)}\right\}^{-1}
$$

بهدست مى آيد. در ادامه، برآورد بيزى پارامتر R تحت تابع زيان توان هاى دوم خطا، وقتى $\theta$ و $\alpha$ متغيرهاى

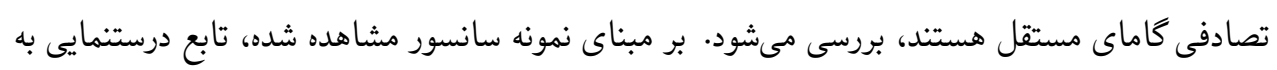

$L($ data, $\beta \mid \theta, \alpha) \propto \theta^{J_{\curlywedge}} \alpha^{J_{\curlyvee}} e^{-\theta V(\beta)-\alpha U(\beta)}$

است. بنابراين تابع جگالى يسين توام بهصورت

$$
\begin{aligned}
& \pi(\theta, \alpha \mid \text { data, } \beta) \propto L(\text { data, } \beta \mid \theta, \alpha) \pi_{\uparrow}(\theta) \pi_{\Upsilon}(\alpha) \\
& \propto \quad \theta^{J_{\curlywedge}} \alpha^{J_{\uparrow}} e^{-\theta V(\beta)-\alpha U(\beta)} \theta^{a_{\uparrow}-1} e^{-b_{\uparrow} \theta} \alpha^{a_{\uparrow}-1} e^{-b_{\uparrow} \alpha} \\
& \propto \quad \theta^{J_{\uparrow}+a_{\uparrow}-1} \alpha^{J_{\uparrow}+a_{\curlyvee}-1} e^{-\theta\left(b_{\uparrow}+V(\beta)\right)-\alpha\left(b_{\curlyvee}+U(\beta)\right)} \\
& =\frac{\left(b_{\uparrow}+V(\beta)\right)^{J_{\uparrow}+a_{\uparrow}}\left(b_{\Upsilon}+U(\beta)\right)^{J_{\Upsilon}+a_{\Upsilon}}}{\Gamma\left(J_{\uparrow}+a_{\uparrow}\right) \Gamma\left(J_{\Upsilon}+a_{\Upsilon}\right)} \\
& \times \quad \theta^{J_{\uparrow}+a_{\uparrow}-1} \alpha^{J_{\curlyvee}+a_{\curlyvee}-1} e^{-\theta\left(b_{\uparrow}+V(\beta)\right)-\alpha\left(b_{\digamma}+U(\beta)\right)},
\end{aligned}
$$

است، كه در آن (B) V( تابع زيان توانهاى دوم خطا از حل انتخرال

$$
\widehat{R}^{B}=\int_{o}^{\infty} \int_{\circ}^{\infty} \frac{\theta}{\alpha+\theta} \pi(\theta, \alpha \mid \text { data, } \beta) d \theta d \alpha
$$

بهدست مىآيد. اكنون با در نظر كرفتن تبديلهاى يك به يك نتيجه گرفت كه ا $>$ ن 


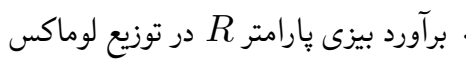

$$
\begin{aligned}
& \text { بنابراين رابطه } \\
& \widehat{R}^{B}=\frac{\left(b_{\uparrow}+V(\beta)\right)^{J_{\uparrow}+a_{\uparrow}}\left(b_{\curlyvee}+U(\beta)\right)^{J_{\Upsilon}+a_{\curlyvee}}}{\Gamma\left(J_{\uparrow}+a_{\uparrow}\right) \Gamma\left(J_{\curlyvee}+a_{\curlyvee}\right)} \\
& \times \int_{0}^{1} \int_{0}^{\infty} u_{\uparrow}^{J_{1}+a_{\uparrow}}\left(1-u_{\uparrow}\right)^{J_{\uparrow}+a_{\uparrow}-1} u_{\uparrow}^{w-1} \\
& \times e^{-u_{\uparrow}\left\{u_{\uparrow}\left(b_{\uparrow}+V(\beta)\right)+\left(1-u_{\uparrow}\right)\left(b_{\uparrow}+U(\beta)\right)\right\}} d u_{\uparrow} d u_{\uparrow} \\
& =\frac{(1-z)^{J_{1}+a_{\curlywedge}}}{B\left(J_{\uparrow}+a_{\uparrow}, J_{\Upsilon}+a_{\Upsilon}\right)} \int_{0}^{1} u_{\uparrow}^{J_{\uparrow}+a_{\uparrow}}\left(1-u_{\uparrow}\right)^{J_{\Upsilon}+a_{\Upsilon}-1}\left(1-u_{\uparrow} z\right)^{-w} d u_{\uparrow}
\end{aligned}
$$

است، كه در آن آن

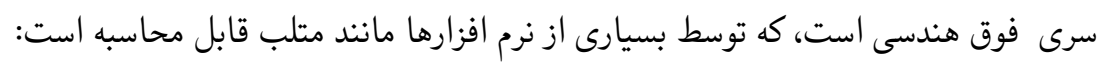

$$
{ }_{r} F_{\backslash}(\alpha, \beta ; \gamma, z)=\frac{1}{B(\beta, \gamma-\beta)} \int_{0}^{1} t^{\beta-1}(1-t)^{\gamma-\beta-1}(1-t z)^{-\alpha} d t,|z|<1 .
$$

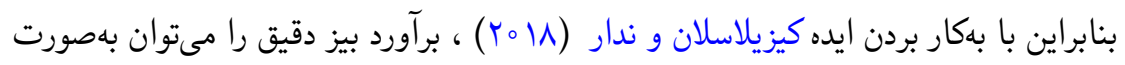

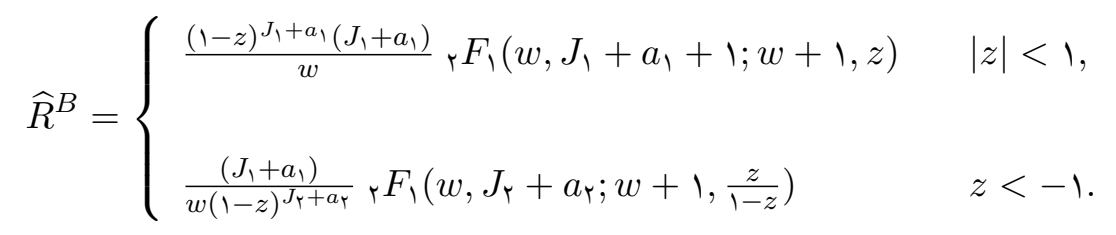

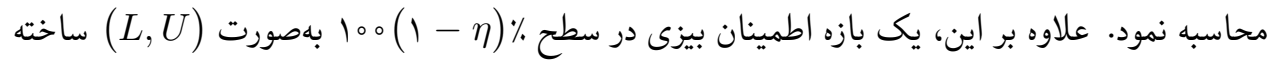
مىشود كه در آن L و ل

$$
\int_{0}^{L} f_{R}(r) d r=\frac{\eta}{r}, \quad \int_{0}^{U} f_{R}(r) d r=1-\frac{\eta}{r}
$$

صدق كنند، كه در نهايت با استفاده از (11) و روش تغيير متغير (r)

$$
f_{R}(r)=\frac{(1-z)^{J_{\uparrow}+a_{\uparrow}} r^{J_{\Upsilon}+a_{\Upsilon}-1}(1-r)^{J_{\uparrow}+a_{\curlywedge}-1}(1-r z)^{-w}}{B\left(J_{\uparrow}+a_{\uparrow}, J_{\curlyvee}+a_{\Upsilon}\right)} .
$$




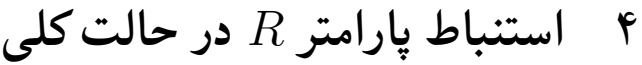

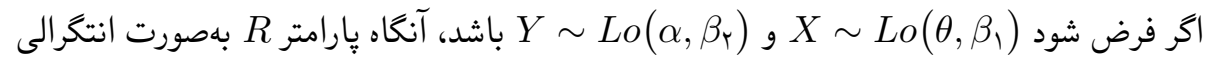

$$
R=1-\alpha \beta_{\curlyvee} \int_{0}^{\infty}\left(1+\beta_{1} y\right)^{-\theta}\left(1+\beta_{\curlyvee} y\right)^{-\alpha-1} d y .
$$

محاسبه مىشود. بر اين مبنا، اكر طرحهاى بهترتيب تابع درستنمايى بر مبناى دادههاى مشاهده شده برابر است با:

$$
\begin{aligned}
& L\left(\mathrm{data} \mid \theta, \alpha, \beta_{\uparrow}, \beta_{\curlyvee}\right) \propto \theta^{J_{\uparrow}} \beta_{\uparrow}^{J_{\uparrow}}\left(\prod_{i=1}^{J_{\uparrow}}\left(1+\beta_{\uparrow} x_{i}\right)^{-1}\right) \alpha^{J_{\curlyvee}} \beta_{\uparrow}^{J_{\top}}\left(\prod_{j=1}^{J_{\curlyvee}}\left(1+\beta_{\curlyvee} y_{j}\right)^{-1}\right) \\
& \times e^{-\theta\left(\sum_{i=1}^{J_{1}}\left(R_{i}+1\right) \log \left(1+\beta, x_{i}\right)+R_{J_{1}}^{*} \log \left(1+\beta_{,} T_{,}\right)\right)} \\
& \times e^{-\theta\left(\sum_{j=1}^{J_{\digamma}}\left(S_{j}+1\right) \log \left(1+\beta_{\Upsilon} y_{j}\right)+S_{J_{\digamma}}^{*} \log \left(1+\beta_{\Upsilon} T_{\digamma}\right)\right)} . \\
& \text { بنابراين برآوردهاى ماكسيم درستمايى } \theta \text { و } \alpha \text { بهترتيب بهفرم } \\
& \widehat{\theta}\left(\beta_{1}\right)=J_{1}\left\{\sum_{i=1}^{J_{1}}\left(R_{i}+1\right) \log \left(1+\beta_{\uparrow} x_{i}\right)+R_{J_{1}}^{*} \log \left(1+\beta_{\backslash} T_{1}\right)\right\}^{-1}, \\
& \widehat{\alpha}\left(\beta_{\Upsilon}\right)=J_{\Upsilon}\left\{\sum_{j=1}^{J_{\digamma}}\left(S_{j}+1\right) \log \left(1+\beta_{\Upsilon} y_{j}\right)+S_{J_{\digamma}}^{*} \log \left(1+\beta_{\Upsilon} T_{\Upsilon}\right)\right\}^{-1},
\end{aligned}
$$

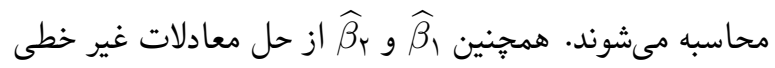

$$
\begin{aligned}
& \frac{J_{1}}{\beta_{\backslash}}-\sum_{i=1}^{J_{1}}\left(\theta\left(R_{i}+1\right)+1\right) \frac{x_{i}}{1+\beta_{\backslash} x_{i}}-\theta R_{J_{1}}^{*} \frac{T_{\backslash}}{1+\beta_{\backslash} T_{\uparrow}}=\circ, \\
& \frac{J_{1}}{\beta_{\Upsilon}}-\sum_{j=1}^{J_{\Upsilon}}\left(\alpha\left(S_{j}+1\right)+1\right) \frac{y_{j}}{1+\beta_{\Upsilon} y_{j}}-\alpha S_{J_{\digamma}}^{*} \frac{T_{\Upsilon}}{1+\beta_{\Upsilon} T_{\Upsilon}}=。 \text {. }
\end{aligned}
$$


ب برآورد بيزى بارامتر R در توزيع لوماكس

$\Delta T_{0}$

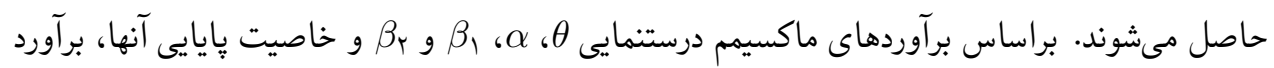
R

$$
\widehat{R}=1-\widehat{\alpha} \widehat{\beta}_{\Upsilon} \int_{0}^{\infty}\left(1+\widehat{\beta}_{1} y\right)^{-\widehat{\theta}}\left(1+\widehat{\beta}_{\Upsilon} y\right)^{-\widehat{\alpha}-1} d y .
$$

حال به برآورد استنباط بيزى پارامتر R تحت تابع زيان توانهاى دوم خطا، با توزيعهاى ييشين

$$
\begin{aligned}
& \pi_{\uparrow}(\theta) \propto \theta^{a_{\uparrow}-1} e^{-b_{\uparrow} \theta}, \theta, a_{\uparrow}, b_{\uparrow}>\circ, \\
& \pi_{\digamma}(\alpha) \propto \alpha^{a_{\digamma}-1} e^{-b_{\digamma} \alpha}, \alpha, a_{\digamma}, b_{\curlyvee}>\circ, \\
& \pi_{\Gamma}\left(\beta_{\uparrow}\right) \propto \beta_{\uparrow}{ }^{a_{\Gamma}-1} e^{-b_{\digamma} \beta_{\uparrow}}, \beta_{\uparrow}, a_{\Gamma}, b_{\Gamma}>0, \\
& \pi_{\digamma}\left(\beta_{\digamma}\right) \propto \beta_{\digamma}^{a_{\digamma}-1} e^{-b_{*} \beta_{\digamma}}, \beta_{\digamma}, a_{\uparrow}, b_{\psi}>0,
\end{aligned}
$$

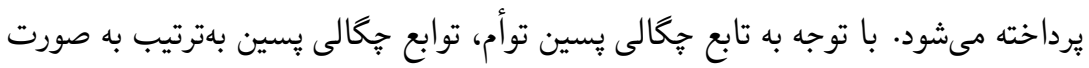

$$
\begin{aligned}
& \theta \mid \beta_{\uparrow}, \text { data } \sim \Gamma\left(J_{\uparrow}+a_{\uparrow}, b_{\uparrow}+V\left(\beta_{\uparrow}\right)\right), \\
& \alpha \mid \beta_{\Upsilon} \text {, data } \sim \Gamma\left(J_{\Upsilon}+a_{\Upsilon}, b_{\Upsilon}+U\left(\beta_{\Upsilon}\right)\right), \\
& \pi\left(\beta_{\uparrow} \mid \theta, \text { data }\right) \propto \beta_{\uparrow}^{J_{1}+a_{r}-1} e^{-\theta\left(V\left(\beta_{\uparrow}\right)+b_{r}\right)} \prod_{i=1}^{J_{\curlywedge}} \frac{1}{1+\beta_{\uparrow} x_{i}}, \\
& \pi\left(\beta_{\digamma} \mid \alpha, \text { data }\right) \propto \beta_{\digamma}^{J_{\digamma}+a_{\digamma}-1} e^{-\alpha\left(U\left(\beta_{\digamma}\right)+b_{\digamma}\right)} \prod_{j=1}^{J_{\digamma}} \frac{1}{1+\beta_{\digamma} y_{j}} .
\end{aligned}
$$

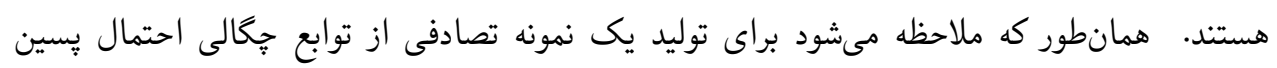
T) بايد از روش مترويوليس-هستينگز استفاده شود.

$$
\begin{aligned}
& \text { الخوريتم بـ برآورد كيبز R: } \\
& \text { كام ا- با مقادير اوليه })
\end{aligned}
$$

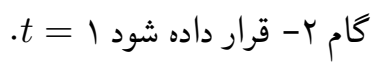

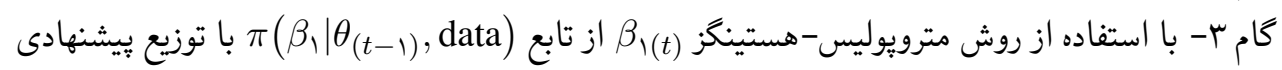

$$
N\left(\beta_{1(t-1)}, 1\right)
$$




$$
\begin{aligned}
& \text { كام ץ- با استفاده از روش مترويوليس-هستينكز } \\
& N\left(\beta_{\Upsilon(t-1)}, 1\right) \\
& \text { كام ها } \\
& \text { كام } \\
& \text { كام }
\end{aligned}
$$

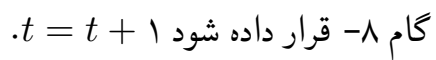

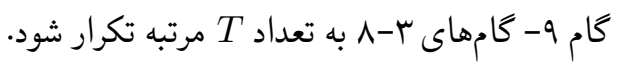

بنابراين برآورد بيزى پارامتر R تحت تابع زيان توانهاى دوم خطا به صورت

$$
\hat{R}^{M C}=\frac{1}{T} \sum_{t=1}^{T} R_{t} .
$$

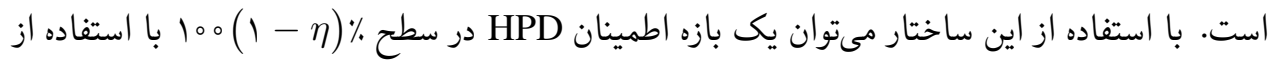
روش جن و شائو (1999) ارائه نمود.

\section{ه مطالعات شبيهسازى}

عملكرد برآوردكرهاى مختلف بيزى، تحت طرحهاى HPC، با استفاده از روش شبيهسازى مونت كارلو

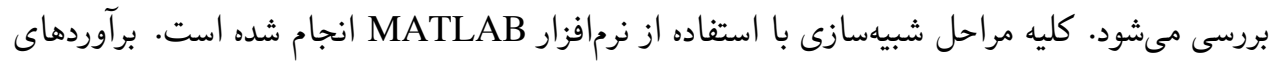

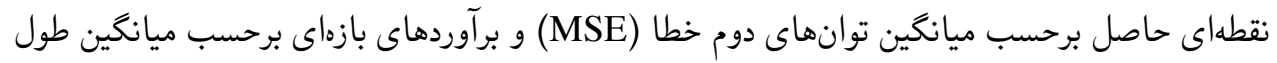

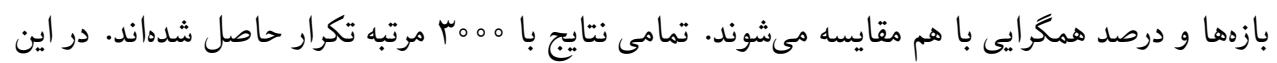

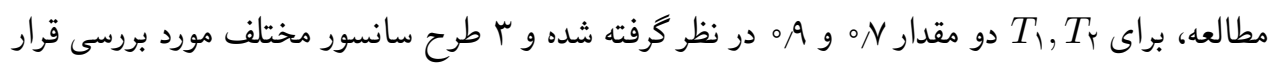

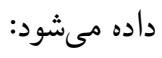

$$
\begin{aligned}
& R_{1}=\ldots=R_{n-1}=\circ, R_{n}=N-n: \text { طرح } \\
& R_{\uparrow}=N-n, R_{\uparrow}=\ldots=R_{n}=\circ:{ }^{r}{ }^{\prime} \\
& R_{1}=\ldots=R_{n}=\frac{N-n}{n}: \text { طرح }
\end{aligned}
$$

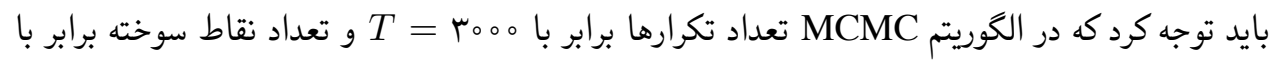

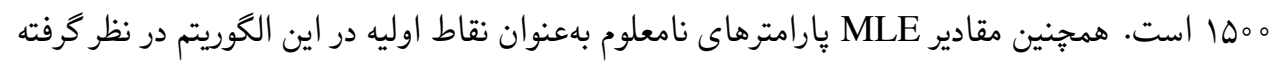


برآورد بيزى بارامتر R در توزيع لوماكس

$$
\begin{aligned}
& \text { شده است. علاوه بر آن، استنباط بيزى با استفاده از توزيعهاى بيشين متفاوتى بهصورت }
\end{aligned}
$$

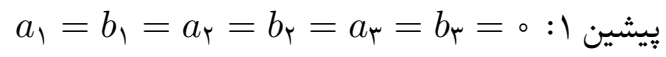

$$
\begin{aligned}
& a_{1}=a_{r}=a_{r}=1, b_{\uparrow}=b_{r}=b_{r}=0,1: r \text { بيشين } \\
& a_{\uparrow}=b_{\uparrow}=a_{\Upsilon}=b_{\Upsilon}=0 \text { بيشين } \\
& a_{1}=a_{r}=1, b_{\uparrow}=b_{r}=0,1: \text { بيشين }
\end{aligned}
$$

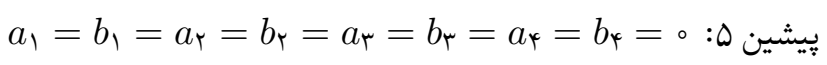

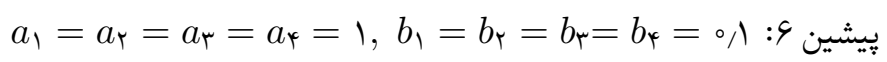

$$
\begin{aligned}
& \text { بررسى شده است. }
\end{aligned}
$$

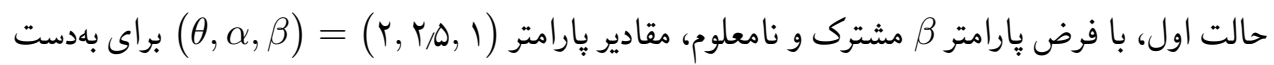

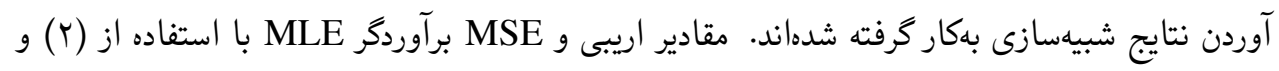

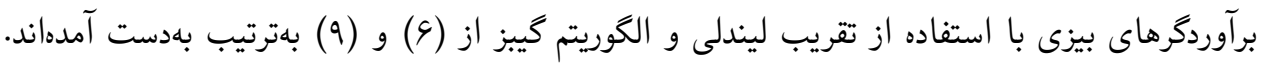

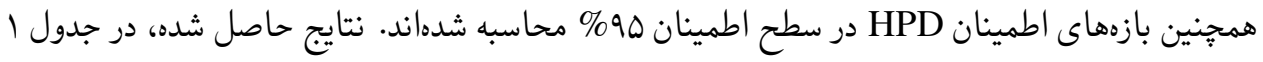
كزارش شده است.

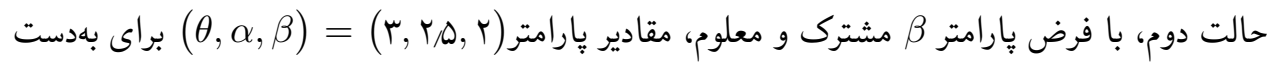

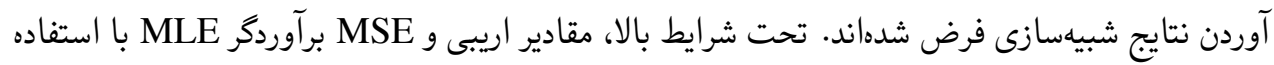

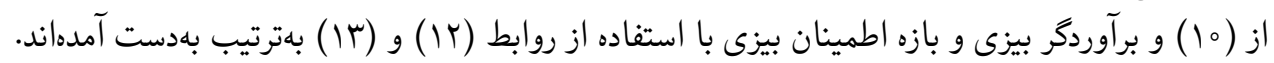
نتايج حاصل شده، در جدول r ارائه شده است.

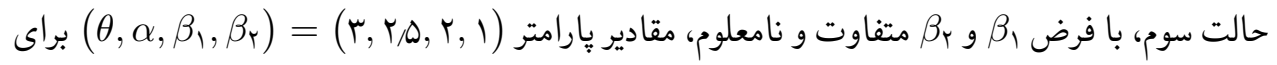

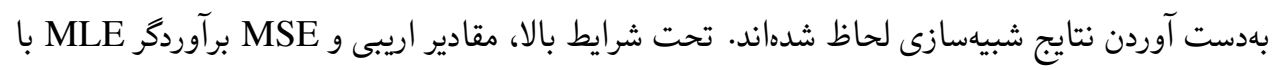

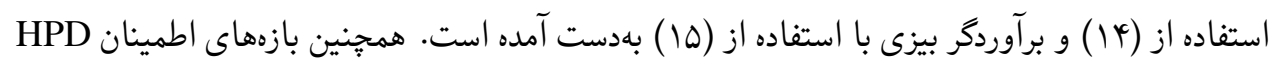
در سطح 90\% محاسبه شدهاند. نتايج حاصل شده، در جدول ب كزارش شده است.

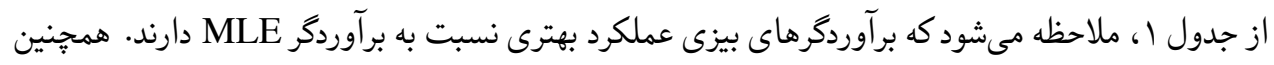

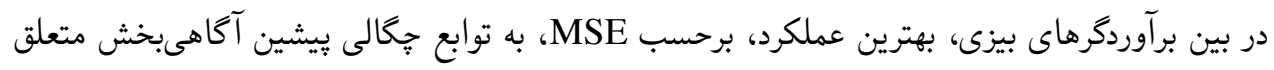

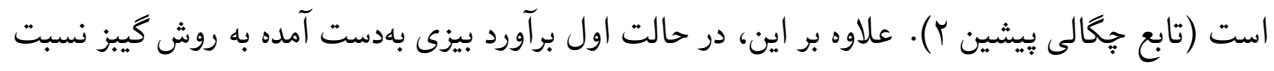

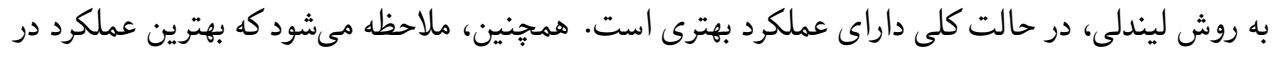

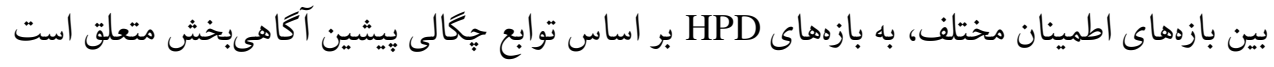




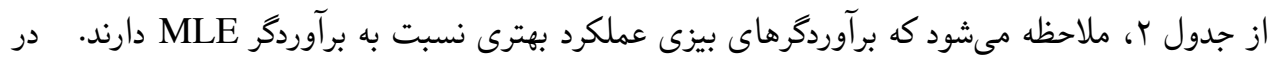

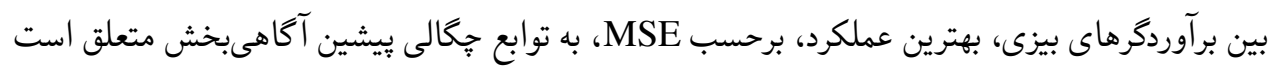

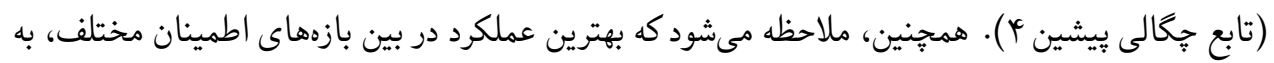

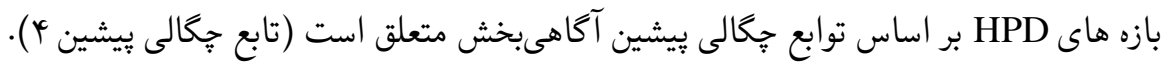

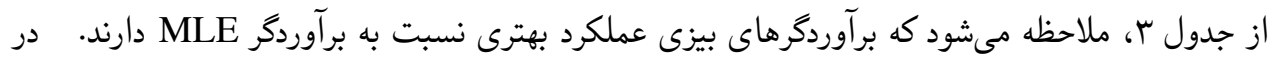

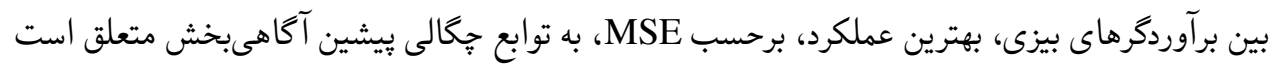

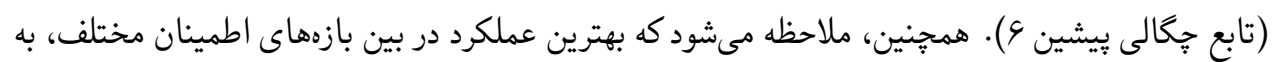

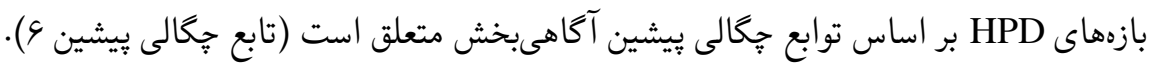

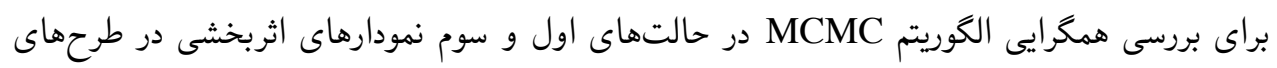

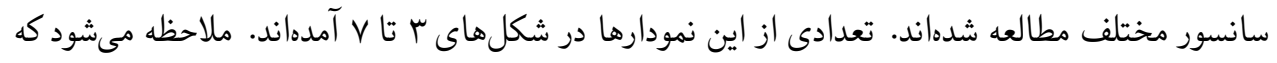

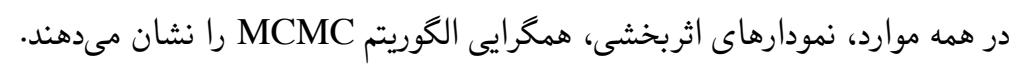

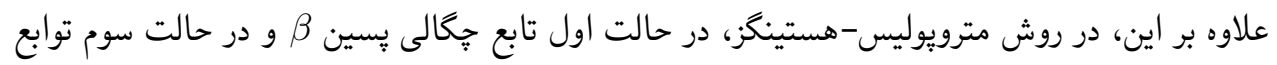

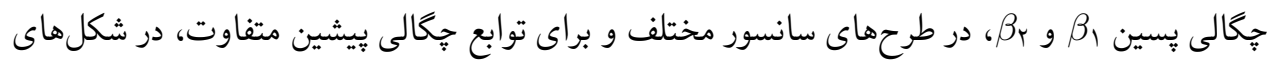

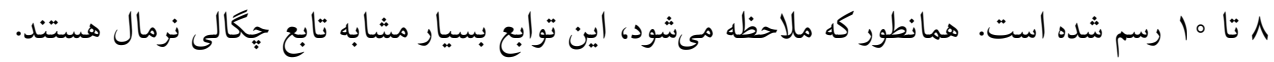
بنابراين براى توليد نمونه تصادفى از

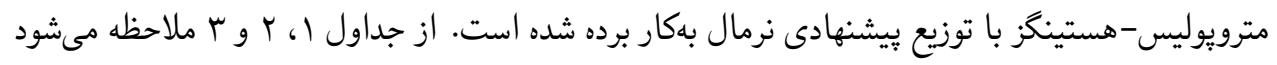

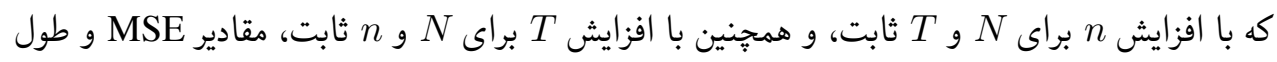

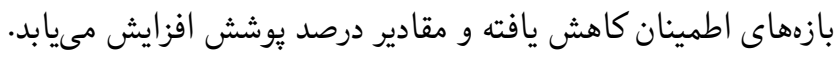


جدول ا. نتايج شبيسازى با پارامتر شكل مشترى و نامعلوم

\begin{tabular}{|c|c|c|c|c|c|c|c|c|c|c|c|c|c|c|c|}
\hline \multicolumn{6}{|c|}{ ي بيشين r } & \multicolumn{6}{|c|}{ ي بيشين 1} & & & \multirow{2}{*}{ C.S } & \multirow{2}{*}{$(N, n)$} \\
\hline MSE & اريبى & C.P & طول & MSE & اريبى & MSE & اريبى & C.P & طول & MSE & اريبى & MSE & اريبى & & \\
\hline.$/ 14$ & $0 / 001$ & $\circ / 90 r$ & -/rar & $0 / 010$ & $0 / 009$ & $0 / 01 V$ & $0 / 009$ & $0 / 900$ & $\circ / 4 \circ 4$ & $0 / 010$ & $0 / 010$ & O/Ort & $0 / 00 \wedge$ & $(1,1)$ & $\left(\psi_{0}, 1_{0}\right)$ \\
\hline$\% 10$ & $0 / 001$ & $\circ / 9 \circ \mathrm{V}$ & $0 / r q 4$ &.$/ 011$ &.$\% 01$ & O/OYI & $\circ \% \circ V$ & $0 / 90 Y$ & $\circ / 4 \circ \varphi^{r}$ &.$/ 011$ & $\circ / 001$ & $0 / 0+9$ & $\circ / 001$ & $(r, Y)$ & $T=\circ / \mathrm{V}$ \\
\hline$\% / 1 T$ & $0 / 009$ & $\circ / 901$ & $\circ / 499$ & $0 / 009$ & $\circ \% \circ \mathrm{V}$ & $0 / 014$ & $0 / 010$ & $\circ / 9 \circ r$ & $\circ / Y \circ Y$ & $0 / 010$ & $\circ / 0 \circ \Lambda$ & O/OYI &.$/ 011$ & $(r, r)$ & \\
\hline $.01 r$ & $0 / 001$ & $0 / 910$ & - / KAS & $0 / 009$ & $0 / 009$ & $0 / 010$ & $0 / 009$ &.$/ 910$ & $0 / r_{0} r$ & $0 / 009$ &.$/ 011$ & O/OTr &.$/ 001$ & $(1,1)$ & $\left(\mu_{0}, 1_{0}\right)$ \\
\hline .111 & $\% / \circ \mathrm{V}$ & $0 / 919$ & $\circ / \% q_{0}$ & $0 / 001$ &.$\% \circ \wedge$ & $0 / 01 k$ & $0 / 011$ &.$/ 910$ & $\circ / F \circ r$ & $0 / 001$ &.$/ 011$ & OOKY & $0 / 011$ & $(r, r)$ & $T=\circ / q$ \\
\hline$\% 11$ & $\circ / \circ \circ \mathrm{V}$ & $\circ / 9 r_{0}$ & $\circ / 790$ & $0 / 009$ & $0 \% 09$ & $0 / 01 r$ & $0 / 011$ & $0 / 911$ & $\circ / 4 \circ \Lambda$ & $0 / 009$ & $\circ / 0 \circ \lambda$ & o/०rI & $0 / 009$ & $(r, r)$ & \\
\hline$\%$ &.$/ 011$ & $\circ / 9 r_{0}^{\circ}$ & $0 / r V I$ & $0 / 0 Y q$ & $0 \% 09$ & $0 / 0 r v$ & $0 / 010$ & $0 / 910$ & $\circ / r V V$ & $0 / 0 T V$ & $0 / 009$ & $\circ / \circ \Delta V$ &.$/ 011$ & $(1, r)$ & \\
\hline $0 / 014$ & $0 / 011$ & $\circ / 9 \mu_{0}$ & $\circ / q_{0}$ & $0 / 009$ & $0 / 009$ & $0 / 019$ & $0 / 010$ & $0 / 949$ & $\circ / 4 \circ \varphi^{f}$ & $0 / 010$ & $0 / 010$ & $0 / 0 T_{1}$ & $0 / 009$ & $(1,1)$ & $(90,10)$ \\
\hline$\% 1 r$ & $0 / 00 \wedge$ & ./qTY & $0 / 4 q 1$ & $0 / 009$ & $\circ \% \circ V$ &.$/ 010$ & $0 / 011$ & O/GTY & $\circ / 4 \circ \varphi^{t}$ & $0 / 010$ & $\circ / 0 \circ V$ & ס/Orr & $0 / 009$ & $(r, r)$ & $T=\circ / q$ \\
\hline $0 \% 04$ & $0 / 010$ & $0 / q y y$ & $\circ / r 90$ & $\circ / \circ \circ \Delta$ & $0 \% 09$ & $0 / 009$ & $0 / 009$ & o/qut & $0 / r q V$ & $\circ / 000$ & $0 / 010$ & $\circ / 001$ & $\% / 00 \wedge$ & $(r, r)$ & \\
\hline$\%$ \% & $\circ / \circ 0 V$ & $\circ / 94_{0}$ & -/TDS & O/OYY & $0 \% 09$ &.$/ \% \Delta$ & $0 / 010$ & $0 / q 4 q$ & $0 / T 40$ & O/OTY & $\circ / \circ \mathrm{V}$ & $\circ / \circ \Delta \Delta$ & $0 / 009$ & $(1, Y)$ & \\
\hline$\%$ & $0 / 001$ &.$/ 990$ & $0 / 199$ & o/oor & $\% \% 0 \mathrm{~V}$ & $0 / 00 \Delta$ &.$/ 011$ &.$/ 900$ & $0 /$ IVte & o/oor & $0 / 009$ & $0 / 004$ & $0 / 001$ & $(1,1)$ & $\left(\varphi_{0}, \mu_{0}\right)$ \\
\hline$\% \% 04$ &.$/ 011$ & $0 / 990$ & $\circ / \mathrm{IVV}$ & \%/or &.$\% 11$ & $0 / 000$ & $0 / 010$ &.$/ 9 \Delta V$ & $0 /|1|$ & $0 / 004$ & $0 / 009$ & $\circ / 001$ & $0 / 011$ & $(r, Y)$ & $T=\circ / q$ \\
\hline$\%$ & $0 / 001$ & $0 / 994$ & $0 / 1 \Delta t$ & o/or &.$\% \circ \wedge$ & $\circ / 000$ & $0 / 009$ & $\circ / 9 \Delta \Delta$ & $\circ / 1 v_{0}$ & o/or & $0 / 001$ & $\circ / \circ \circ V$ & $\circ / \circ \circ V$ & $(r, r)$ & \\
\hline$\%$ & $0 / 009$ & $0 / 990$ & سזו/ס & $0 / 01 k$ & $\% \% 09$ & ./OTD & $0 / 010$ &.$/ 9 \Delta V$ & $0 / \mid \uparrow \Lambda$ & $0 / 0 r_{0}$ & $0 / 011$ & $\circ / \circ \Delta \circ$ & $0 / 010$ & $(1, r)$ & \\
\hline$\%$ & $0 / 010$ &.$/ 991$ & o/ Mkr & o/OOr &.$/ 001$ & $0 / 004$ & $\circ / 00 V$ & $0 / 994$ &.$/ 101$ & o/oor &.$/ 011$ &.$/ 00 \Delta$ & $0 / 010$ & $(1,1)$ & $\left(\Lambda_{0}, \psi_{0}\right)$ \\
\hline$\% \%$ & $0 / 010$ & $0 / 999$ &.$/ 100$ & o/or &.$\% 10$ & o/oor & $\circ / 001$ & $0 / 99 V$ & $\circ / 109$ & \%/ \% r & $0 / 00 \wedge$ & $\circ / 000$ & $\% / 00 \mathrm{~V}$ & $(r, Y)$ & $T=\circ / q$ \\
\hline$\%$ & $\% / \circ \mathrm{V}$ &.$/ 990$ &.$/ 1 \mu \Lambda$ & OOOY & $0 / 009$ & $0 / 004$ & $0 / 001$ &.$/ 9 G 4$ & $0 / 14 q$ & o/or & $0 / 009$ & $0 / 009$ & $\circ / \circ 0 V$ & $(r, r)$ & \\
\hline $0 / 014$ & $0 / 010$ & $0 / 99 V$ & $0 / 1 m T$ & $0 / 010$ & $0 \% 10$ & $0 / 019$ & $0 / 009$ & $0 / 994$ & $0 / 1 \mathrm{kr}$ & $0 / 01 r$ & $0 / 009$ & $0 / 04 y$ & $0 / 011$ & $(1, r)$ & \\
\hline
\end{tabular}


جدول r. نتايج شبيهسازى با پارامتر شكل مشترك و معلوم

\begin{tabular}{|c|c|c|c|c|c|c|c|c|c|c|c|}
\hline \multicolumn{4}{|c|}{ بيشين } & \multicolumn{4}{|c|}{ بيشين r } & \multicolumn{2}{|c|}{ MLE } & \multirow[t]{2}{*}{ C.S } & \multirow[t]{2}{*}{$(N, n)$} \\
\hline C.P & طول & MSE & اريبي & C.P & طول & MSE & اريبى & MSE & اريبي & & \\
\hline o/arY &.$/ 490$ & $\% / \circ \mathrm{V}$ & $0 / 010$ & . /qrr & $\circ / F \circ V$ &.$/ 011$ &.$/ 001$ &.$/ 019$ & .1009 & $(1,1)$ & $(4 \circ, 1 \circ)$ \\
\hline.$/ 940$ &.$/$ rqu & $0 \% 04$ & .1010 &.$/ 919$ & $\circ / 4 \circ \Delta$ &.$\% 11$ & $0 / 010$ & .1018 & $\% \circ \mathrm{V}$ & $(Y, Y)$ & $T=\circ / \mathrm{V}$ \\
\hline$\circ / 9 \circ 0$ &.$/ 499$ & $\% \% \circ V$ & $0 / 00 \wedge$ & $0 / 90 \%$ & $\circ / 4 \circ V$ & $\% \% 1 r$ & $0 / 009$ &.$\% 1 V$ & $\% 10$ & $(r, r)$ & \\
\hline.$/ 904$ &.$/$ rVG & $\%$ \% & $0 / 009$ &.$/ 901$ & $0 /$ rVG & $\% \% r$ & $0 / 009$ & $\% \Delta r$ &.$\% 09$ & $(1, r)$ & \\
\hline.$/ 919$ &.$/ r q 4$ & .1004 &.$/ 010$ &.$/ 910$ & $\circ / 4 \circ \varphi^{\prime}$ & $.01 r$ & $0 / 010$ & .1019 & .1010 & $(1,1)$ & $(4 \circ, 1 \circ)$ \\
\hline ./aro &.$/ 4 q 4$ & $0 \% 04$ & $\circ / \circ \circ V$ & ./ark & $\circ / 4 \circ r^{\prime}$ & .1010 & $0 / 010$ &.$\% 10$ & $\% 10$ & $(Y, Y)$ & $T=\circ / 9$ \\
\hline ./9ro &.$/ 490$ & $0 \% 04$ & $0 / 009$ &.$/ 94 x$ & $0 / 4 \circ 9$ &.$\% 11$ & $0 / 010$ & .1019 & $\% 1 \%$ & $(r, r)$ & \\
\hline ./वrq &.$/ r V r$ & $\%$ & $0 / 010$ &.$/ 9 T \Delta$ & $0 / r v i$ & $\%$ & $0 \% 10$ & $\% \% \wedge$ & $\% \% 09$ & $(1, r)$ & \\
\hline.$/ 9 Y 4$ &.$/ 490$ & $0 / 004$ & $0 / 001$ &.$/ 9 M Y$ & $0 / 4 \circ 9$ & .1011 &.$/ 009$ & .1019 & .1001 & $(1,1)$ & $(9 \circ, 10)$ \\
\hline ./9ro &.$/ 490$ & $0 \% 04$ & $\circ / \circ \circ$ & ./aYk & $0 / 4 \circ y^{\prime}$ &.$\% 11$ &.$/ 00 \wedge$ & .1018 & $\% \%$ & $(Y, Y)$ & $T=\circ / q$ \\
\hline.$/ a Y Y$ &.$/$ rq4 & $0 \% 04$ & $0 / 010$ & ./9rr & $\circ / 4 \circ \Delta$ &.$\% 11$ & $0 / 00 \wedge$ &.$\% 10$ & $\% \% \circ 9$ & $(r, r)$ & \\
\hline.$/ 9 M Y$ & $\circ / r \gamma_{0}$ & $\%$ & $0 / 009$ & r ra / & $0 / r v 1$ & ו & $\% \% 01$ & $\% \circ 01$ & $\% \% 09$ & $(1, r)$ & \\
\hline 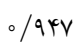 & ./rar &.$/ 000$ &.$/ 010$ &.$/ q k y$ &.$/ 49 V$ & .1004 & $0 / 010$ &.$/ 011$ & .1009 & $(1,1)$ & $\left(\varphi \circ, r_{0}\right)$ \\
\hline.$/ 94 \lambda$ & ./rar & $\% \% 0 \psi^{4}$ & $0 / 00 \wedge$ &.$/ 9 k 4$ & -/ rav &.$\% 04$ &.$/ 011$ &.$\% 09$ & $\% \% \circ$ & $(Y, Y)$ & $T=\circ / q$ \\
\hline.$/ 944$ & ./rar & $\% \% \circ y^{4}$ &.$/ 011$ &.$/ 9 k \mu$ & -/ Tav &.$\% \circ 0$ & $\circ / \circ \circ V$ &.$\% 09$ & $\% 10$ & $(r, r)$ & \\
\hline.$/ 949$ &.$/ K 41$ & $\% \%+1$ & $\circ / \circ \circ V$ &.$/ 949$ & $0 / 491$ &.$\% \pi r$ & $0 / 010$ & $0 \% k r$ &.$\% 09$ & $(1, Y)$ & \\
\hline.$/ 994$ &.$/ 1 v_{0}$ & $\% / 0 \% t$ & .011 &.$/ 909$ & - / IVo &.$/ 004$ & .1010 & $\% / \circ \gamma$ &.$\% 01$ & $(1,1)$ & $\left(\varphi \circ, r_{0}\right)$ \\
\hline.$/ 999$ &.$/ 199$ & $\% \%$ & $0 / 010$ &.$/ 994$ & $0 /$ IVT & $\% \% \circ \psi^{k}$ & $0 / 010$ & $\% \% \circ V$ & $\% 1 \%$ & $(Y, Y)$ & $T=\circ / 9$ \\
\hline.$/ 990$ &.$/ 1 v^{\circ}$ & $\%$ \% r & $\circ / 00 \wedge$ &.$/ 990$ &.$/ 1 v 9$ & $\% \circ \circ \Delta$ &.$/ 011$ &.$\% \circ \wedge$ & $\% \circ \mathrm{V}$ & $(r, r)$ & \\
\hline.$/ 999$ &.$/ 100$ &.$\% 10$ & $0 / 010$ &.$/ 994$ & $0 / 194$ & $\%$ \% ५० & $0 / 010$ &.$\% 49$ & $\% 10$ & $(1, Y)$ & \\
\hline.$/ 999$ &.$/ 14 q$ & \%o०r &.$/ 00 \wedge$ &.$/ 991$ & ./lor & \%or &.$/ 009$ & .1004 & .1011 & $(1,1)$ & $\left(\Lambda_{\circ}, \varphi_{\circ}\right)$ \\
\hline.$/ 941$ &.$/ 149$ & $\% \% O r$ &.$/ 011$ &.$/ 999$ &.$/ 101$ & $\% \circ \%$ & $0 / 00 \wedge$ & .1004 & $\% 1 \%$ & $(Y, Y)$ & $T=\circ / q$ \\
\hline.$/ 9 \gamma_{0}$ &.$/ 1 \psi_{0}$ & $\%$ \%Or &.$\% 01$ &.$/ 991$ & - / ira & $\% \% \circ r$ & $0 / 009$ & $0 \% 04$ & $\% 11$ & $(r, r)$ & \\
\hline$\circ / 9 \gamma_{0}$ & $0 / I r v$ & $\% 10$ &.$/ 010$ &.$/ 991$ & $\circ / / \varphi_{0}$ & .1019 & $\% 11$ & \% & $\% 11$ & $(1, r)$ & \\
\hline
\end{tabular}


جدول r. نتايج شبيهازى در حالت كلى

\begin{tabular}{|c|c|c|c|c|c|c|c|c|c|c|c|}
\hline \multicolumn{4}{|c|}{ بيشين 9} & \multicolumn{4}{|c|}{ بيشين ه } & \multicolumn{2}{|c|}{ MLE } & \multirow[t]{2}{*}{ C.S } & \multirow[t]{2}{*}{$(N, n)$} \\
\hline C.P & طول & MSE & اريبى & C.P & طول & MSE & اريبى & MSE & اريبي & & \\
\hline.$/ 904$ &.$/ r V r$ & 01004 &.$/ 001$ & $0 / 90$ & . &.$/ 001$ & $\% / 001$ &.$/ 01 r$ & .1010 & $(1,1)$ & $(k \circ, 1 \circ)$ \\
\hline.$/ 919$ & . &.$\% 01$ & $\% \% 09$ &.$/ 91 V$ &.$/ 490$ & $0 \% 04$ &.$\% 09$ &.$\% 1 r$ & $\% \% 01$ & $(r, r)$ & $T=\circ / \mathrm{V}$ \\
\hline.$/ 910$ &.$/ r \Lambda 1$ & .1004 &.$\% 09$ & $0 / 904$ & - / rq4 & $\% \% 01$ & $\% \% 9$ & r & $\% \circ 9$ & $(r, r)$ & \\
\hline.$/ 911$ & . & $\%$ Y & 010 &.$/ 914$ & . & $\% \%$ Y & $\% \% 9$ & $\%$ & $\% 19$ & $(1, Y)$ & \\
\hline.$/ 9 Y q$ & $. / r r \mid$ & $\circ / 000$ &.$/ 009$ & ./qYK &.$/$ rqr & $\circ / \circ \%$ &.$/ 009$ & .1010 &.$\% \circ 9$ & $(1,1)$ & $(k \circ, 1 \circ)$ \\
\hline.$/ 9 r q$ & . TAY & $\% \circ \circ \Delta$ & $\% \% \circ \wedge$ &.$/ 919$ &.$/$ rq4 & $\% \circ \circ \Delta$ &.$\% 09$ & $\% 10$ & $\% 1 \%$ & $(r, Y)$ & $T=\circ / 9$ \\
\hline.$/ 94 q$ &.$/ \mu^{\prime}$ &.$\% \circ \Delta$ & $0 / 009$ & ./ATr &.$/ r q 4$ & $\circ \% \circ V$ & .10 &.$/ 011$ & $\% 10$ & $(r, r)$ & \\
\hline ./qrq &.$/ F Y 4$ &.$\% 1 V$ & $0 \% 09$ & ०/940 & ./TOH & $\% \% r 1$ & $\% \% \circ 9$ & $\%$ & $\% 10$ & $(1, r)$ & \\
\hline.$/ q 4 q$ &.$/$ M $_{1}$ & $0 \% 04$ & $\% / 001$ &.$/ 9 T Y$ &.$/ 490$ & $0 / 009$ & $\% / 001$ &.$/ 011$ & $\% / 001$ & $(1,1)$ & $(9 \circ, 10)$ \\
\hline ./949 &.$/ T V \Lambda$ & $\% \% \circ \Delta$ & $0 \% 10$ &.$/ 949$ & . & $\circ \% \circ V$ & $\% 1 \%$ &.$\% 1 r$ & $\% 10$ & $(r, Y)$ & $T=\circ / 9$ \\
\hline.$/ 9 Y \Lambda$ &.$/ r V V$ & $\% \% \circ t^{t}$ & $\% \circ 01$ &.$/ 949$ &.$/$ r I & $0 \% 04$ & .1009 & $0 \% 09$ & $\% \circ \wedge$ & $(r, r)$ & \\
\hline.$/ 949$ &.$/ \% 40$ & $\%$ \% ५ & $\% \% 09$ &.$/ 91 \mathrm{~V}$ &.$/$ rqV & $\%$ Y Q &.$\% 09$ &.$\% 49$ & $\%$ & $(1, r)$ & \\
\hline.$/ 94 \Delta$ & $\circ /$ TAS & $\% / 004$ & $0 / 009$ &.$/ 9 k \mu$ & o/rar & $\% / \circ{ }^{f}$ &.$\% \circ \Lambda$ &.$/ 001$ & $\% / \circ \wedge$ & $(1,1)$ & $\left(\varphi_{0}, r_{\circ}\right)$ \\
\hline.$/ q 4 V$ &.$/$ TAF & $\% \% \circ x^{4}$ &.$\% 0 \wedge$ &.$/ 94 \Delta$ &.$/ 4 \wedge 9$ & $\% \% \circ \psi^{4}$ & $\% 10$ &.$\% 09$ & $\% \% 09$ & $(r, r)$ & $T=\circ / q$ \\
\hline.$/ 949$ &.$/ T A V$ & $\% \% 04$ & $0 \% 09$ &.$/ 9 k 4$ & . /rar & $\% \% \circ 4$ & $0 \% 09$ & $\circ \% \circ \wedge$ & $\% \% \propto \Lambda$ & $(r, r)$ & \\
\hline.$/ 90 \mathrm{r}$ & . / TOV &.$\% 19$ & $0 \% 09$ &.$/ 9 k 4$ &.$/ T O \Lambda$ & $\%$ & $\% 10$ & $\%$ \% H & $\% 11$ & $(1, Y)$ & \\
\hline.$/ 990$ &.$/ 111$ & $\% / 0 \%$ & $0 / 009$ & $\circ / 9 \Delta V$ & $0 / 4 \circ 9$ & $\% / 0 \%$ & .009 & $0 / 004$ &.$/ 009$ & $(1,1)$ & $\left(\varphi_{\circ}, \mu_{\circ}\right)$ \\
\hline.$/ 999$ &.$/ 189$ & $\%$ &.$\% 09$ &.$/ 940$ &.$/ Y I 1$ & $\%$ & $\% \circ \wedge$ & $0 \% 04$ & $\% \% 09$ & $(r, r)$ & $T=\circ / q$ \\
\hline.$/ 999$ & . MAr & $\% \% O r$ &.$\% 09$ & $\circ / 9 \Delta \Delta$ & $O / T \circ T^{\prime}$ & $\% \% \circ y$ &.$\% 09$ & $\% \% \circ D$ & $\% \% 09$ & $(r, r)$ & \\
\hline.$/ 99 V$ & $\circ /|V|$ & 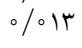 & 010 & $\circ / 901$ &.$/ 194$ &.$\% 19$ & $\% \circ 1$ & $\%$ \% ० & $\% 11$ & $(1, r)$ & \\
\hline.$/ 991$ &.$/$ / & \% o०r &.$/ 009$ &.$/ 994$ &.$/ 100$ & $\% \circ \%$ & $.1 \%$ & $\% / 0 \%$ &.$\% \circ 9$ & $(1,1)$ & $\left(\Lambda \circ, \varphi_{\circ}\right)$ \\
\hline.$/ 9 \vee_{0}$ & & $\% \% 0 r$ & $0 / 009$ &.$/ 99 \mathrm{~V}$ &.$/ 101$ & $\% \% O r$ & $\% \% \wedge$ & $\% \% \circ x^{\prime}$ & $\% 10$ & $(r, r)$ & $T=\circ / 9$ \\
\hline.$/ 999$ & $\circ / 14 \circ$ & $0 \% 0 Y$ & $\circ / 001$ &.$/ 991$ &.$/ 190$ & \%०० &.$\% 09$ & $\% \circ 0 \Delta$ & $\% \circ \wedge$ & $(r, r)$ & \\
\hline.$/ 999$ &.$/ 1 r_{0}$ & $0 / 009$ & $0 / 009$ &.$/ 999$ &.$/ 100$ &.$\% 11$ & .10 &.$/ 10$ & $\%$ & $(1, r)$ & \\
\hline
\end{tabular}




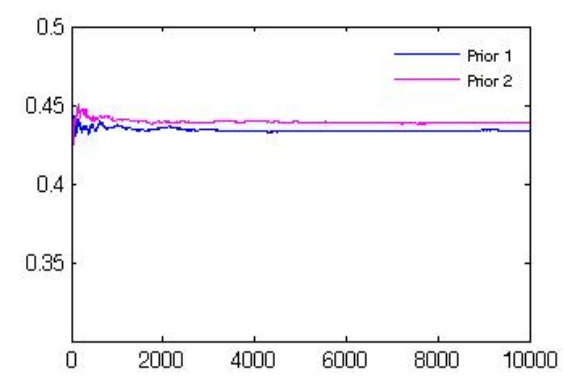

(ب)

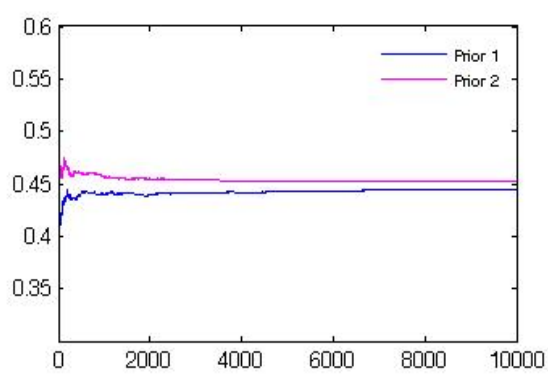

(الف)

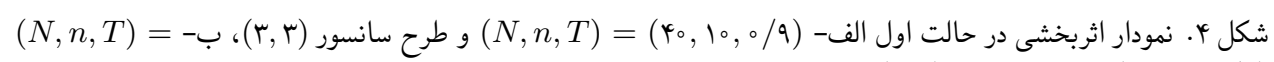

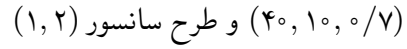

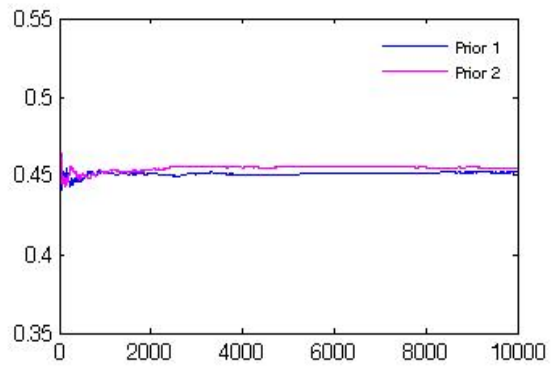

(ب)

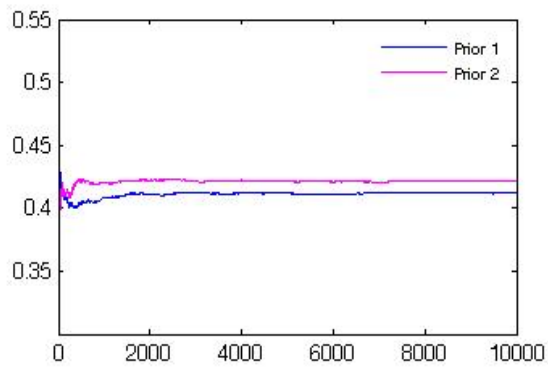

(الف)

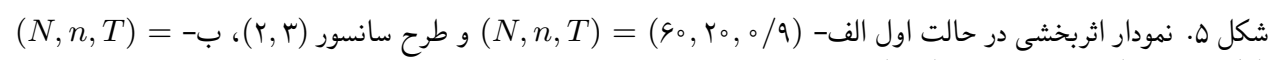
( $)$ و طرح سانسور (1, ro, ro/V)

\section{9 تحليل داده واقعى}

دادههاى اين اين بخش مربوط به دو گروه هستند كه از بيمارى سرطان رنج مىبرند و اولين بار در افرون

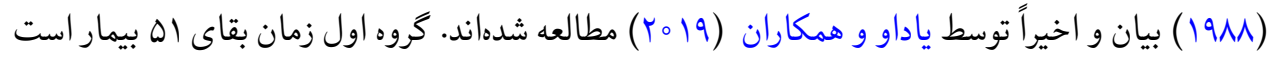

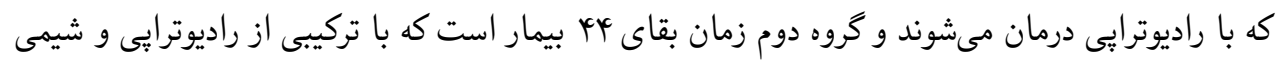

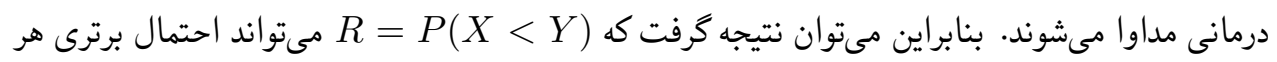

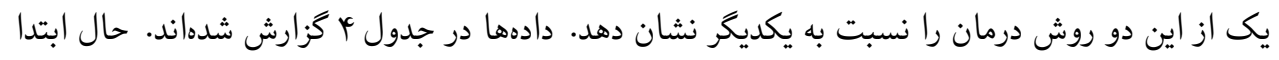




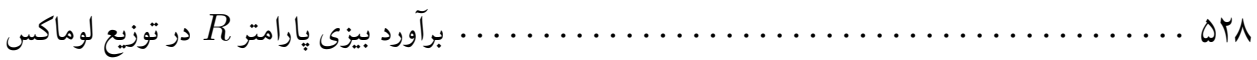

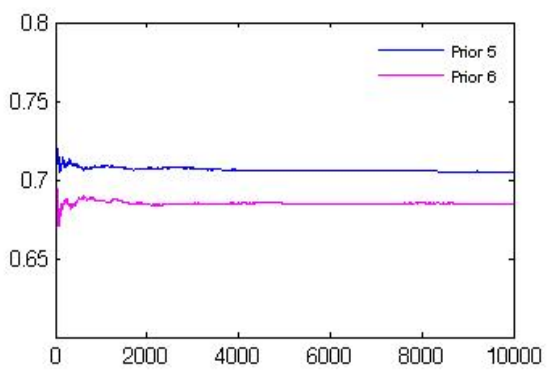

(ب)

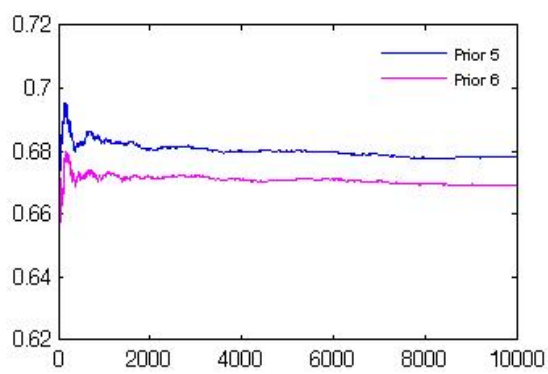

(الف)

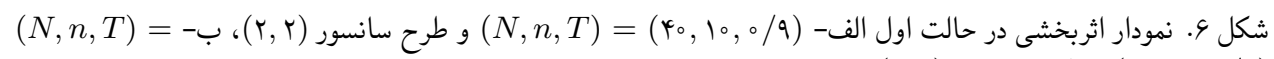

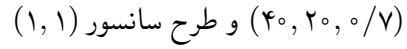

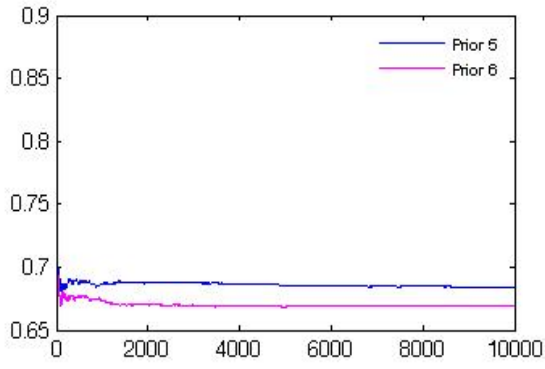

(ب)

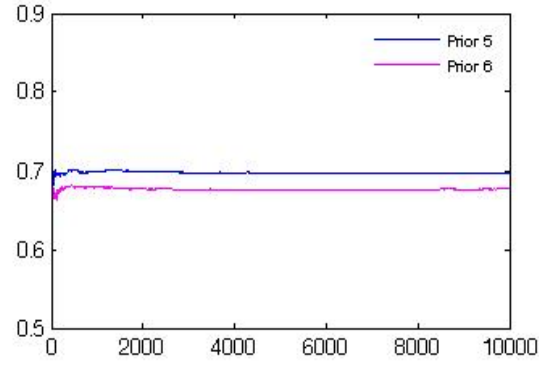

(الف)

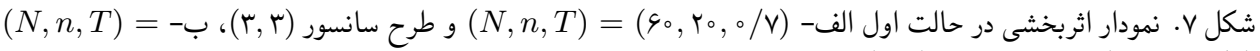

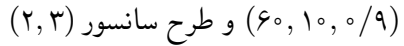

بايد بررسى كرد كه توزيع لوماكس براى تحليل اين دادهها مناسب است، كه براى اين منظور بايد توزيع

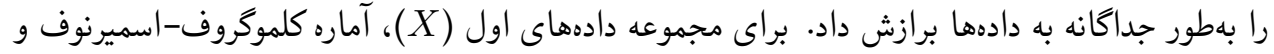

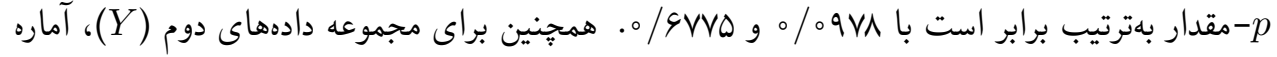

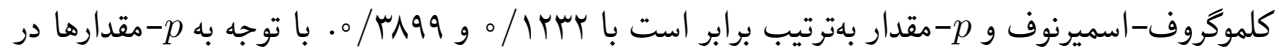

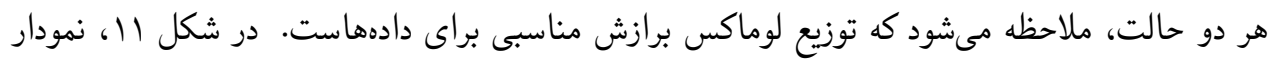

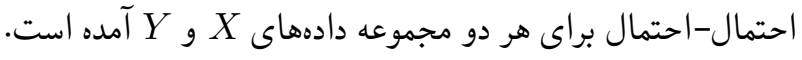

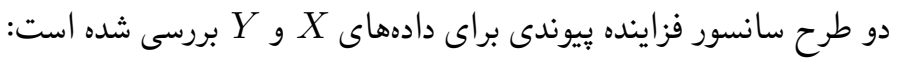
طرح ا، براى 


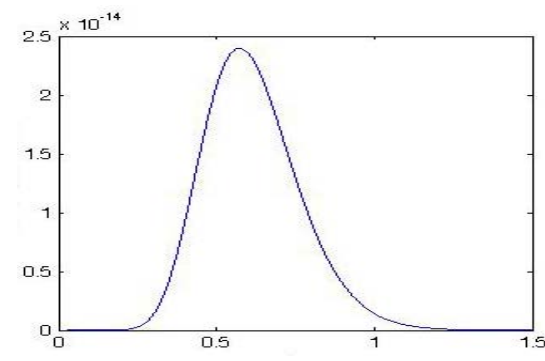

(ب)

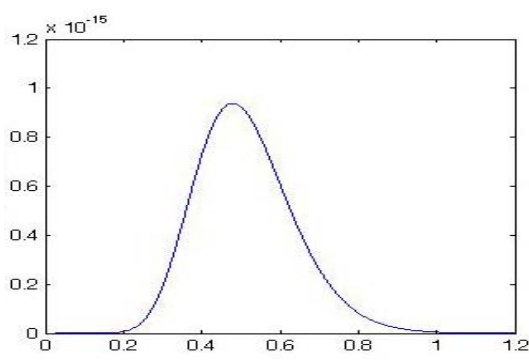

(الف)

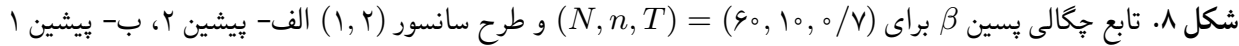

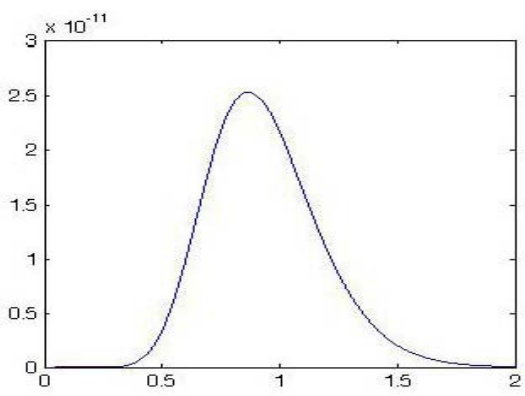

(ب)

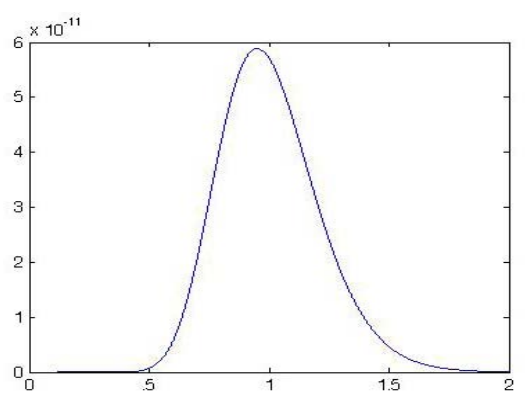

(الف)

شكل 9.

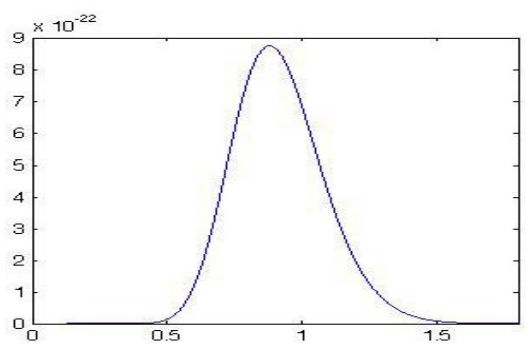

(ب)

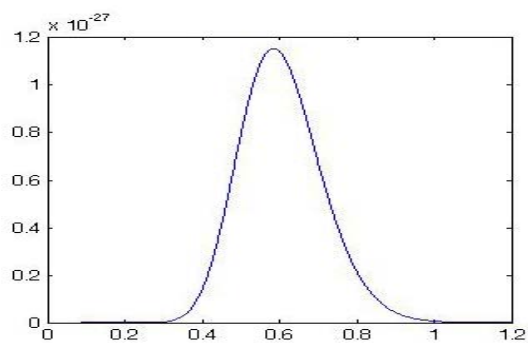

(الف)

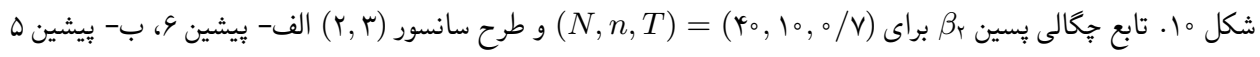


\%

\begin{tabular}{|c|c|c|c|c|c|c|c|c|c|c|}
\hline \multicolumn{11}{|c|}{ جدول F. دادههاى واقعى } \\
\hline$r r / T \Lambda$ & kr & $+1 / \Delta \Delta$ & ry & $r r / v$ & $19 / 1$ & $\mid \psi / \psi \wedge$ & $10 / 4 r$ & v & q/Or & \multirow{6}{*}{$X$} \\
\hline 179 & $11 \%$ & 101 & 91 & $\Lambda^{F}$ & $\Delta r$ & 94 & gr & $\Delta r / g r$ & $4 q / q$ & \\
\hline 190 & $10 \mathrm{~V}$ & lot & 149 & 149 & $1 \psi_{0}$ & $1 t_{0}$ & 179 & Irr & זrו & \\
\hline TrV & rVr & TYA & KYY & rTD & KMA & IVG & IVr & 190 & 190 & \\
\hline \multirow[t]{2}{*}{1144} & 1101 & $\Delta q Y$ & $\Delta \wedge r$ & $\Delta T H$ & $k \psi_{0}$ & Kro & FIV & $Y \circ D$ & rqV & \\
\hline & & & & & & & & & $|4| V$ & \\
\hline$\Delta \Lambda / R G$ & $\Delta \Delta / \ll \varphi$ & 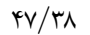 & $\psi i / r \Delta$ & rv & $M I / 9 \Lambda$ & $r \Delta / \wedge V$ & $r r / V{ }^{*}$ & $r r / \Delta Q$ & $\mid r / T_{0}$ & \multirow{5}{*}{$Y$} \\
\hline 110 & 94 & 95 & $\Delta 4$ & kr & 1) & $V \varphi / k v$ & $V \wedge / K^{Q}$ & $9 \Lambda / 4 G$ & $G r / F V$ & \\
\hline IVr & 109 & 100 & 149 & 140 & זrו & $\mu_{0}$ & ITV & 119 & $11 r$ & \\
\hline \multirow[t]{2}{*}{019} & 149 & Frt & & 419 & TAl & YKq & roq & 194 & 189 & \\
\hline & & & & & & 1679 & NIV & Vro & & \\
\hline
\end{tabular}

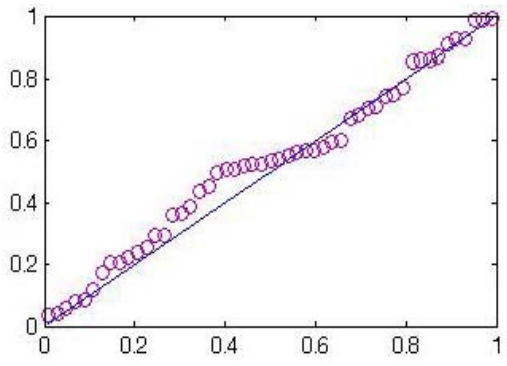

(ب)

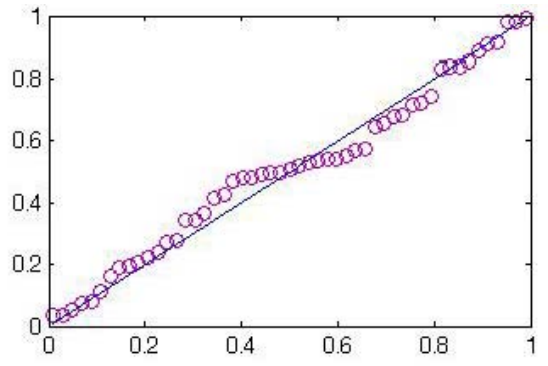

(الف)

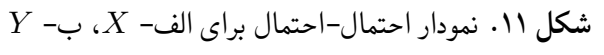

$$
\text { طرح r، براى }
$$

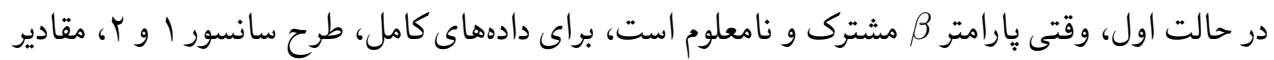

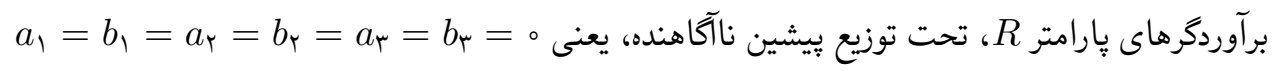

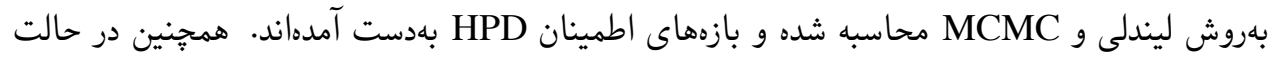

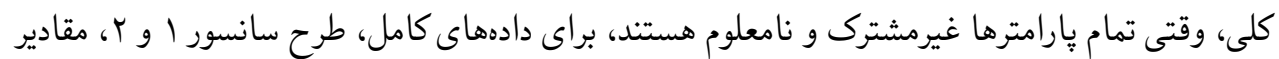

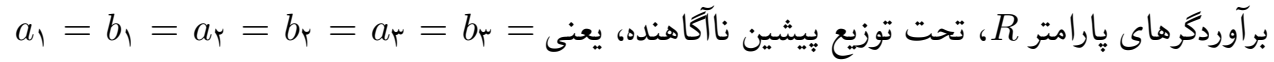

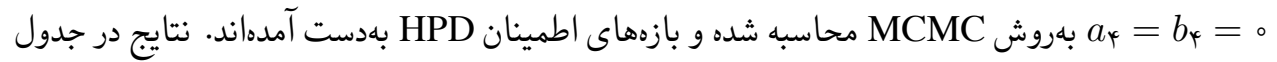

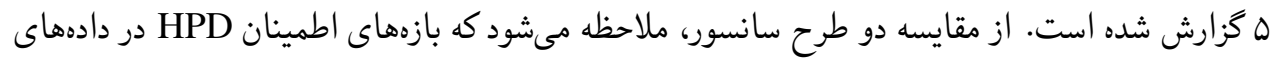

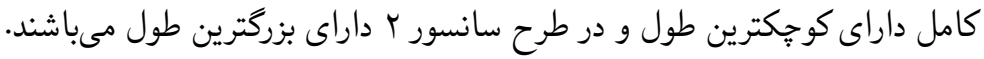




\begin{tabular}{|c|c|c|c|c|c|c|c|}
\hline \multicolumn{8}{|c|}{ جدول ه. نتايج در دادههاى واقعى } \\
\hline \multicolumn{3}{|c|}{ 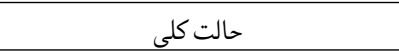 } & \multicolumn{4}{|c|}{ حالت اول } & \multirow[b]{2}{*}{ طرح } \\
\hline HPD & كيبز & MLE & HPD & ليندلى ل & كيبز & MLE & \\
\hline$(\circ / r V T, \circ / 9 \Delta r)$ &.$/ 4 \wedge 9$ &.$/ 4 \lambda 1$ & $(\circ / 4 \circ 9, \circ / 94 \Delta)$ & . /Orr & .019 & .019 & كامل \\
\hline$(0 / r \Delta 1,0 / 9 \wedge \Lambda)$ & . KAT & $\circ / 4 \lambda I$ & $(\circ /$ $/ \mu, \circ / 99 Y)$ & $\circ / \Delta T_{1}$ &.$/ 01 \%$ &.$/ 01 \%$ & 1 \\
\hline$\left(\circ / T_{T \Lambda}, \circ / \mathrm{V} \circ \mathrm{V}\right)$ &.$/ 01 \%$ &.$/ 011$ & $(0 /$ TMG, ०/VKr $)$ &.$/ \Delta A Y$ & . /orv & ./DT & r \\
\hline
\end{tabular}

\section{بحث و نتيجهگيرى}

در اين مقاله، تحت سانسور فزاينده بيوندى، زمانىكه متغيرهاى تنش و مقاومت داراى توزيع لوماكس

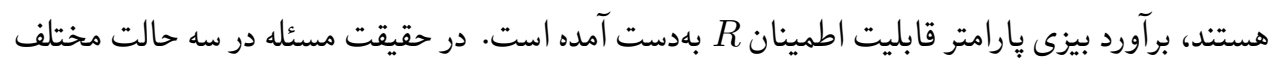

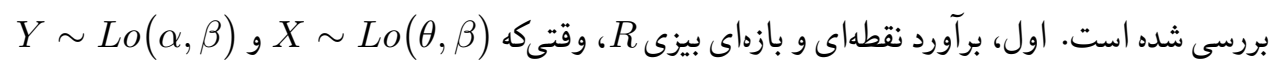

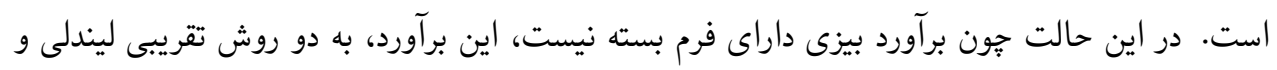

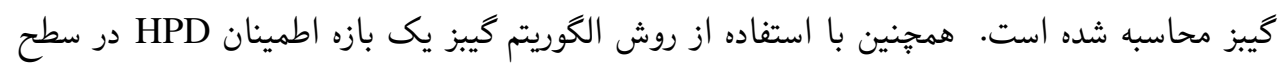

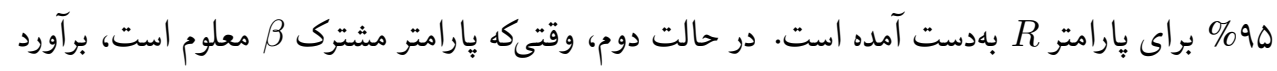

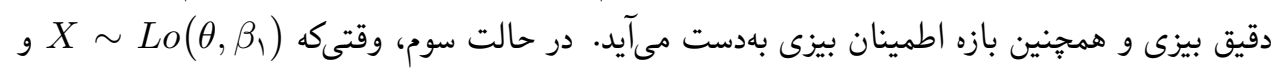
نتابح

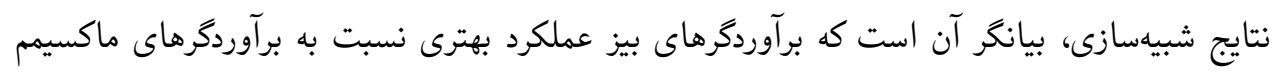

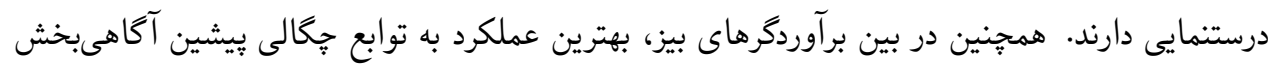

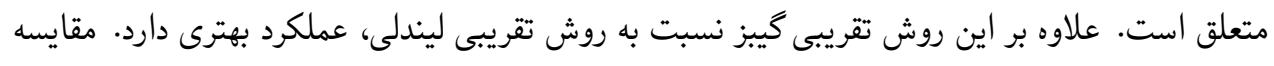

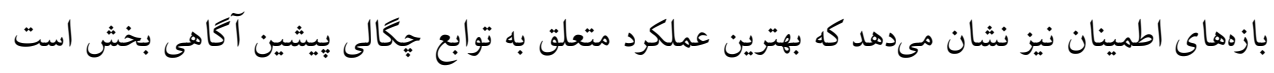

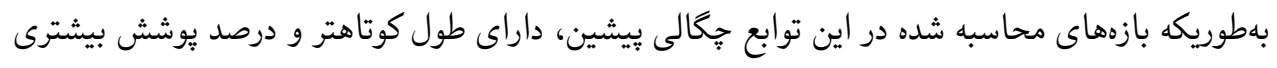

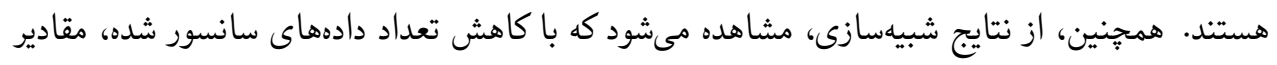
MSE

\section{تقدير و تشكر}

نويسندكان از داوران مقاله، ويراستار و سردبير محترم مجله كه با نظرات ارزشمند خود در بهبود كيفيت مقاله نقش مهمى داشتهاند، سياسكزارند. 


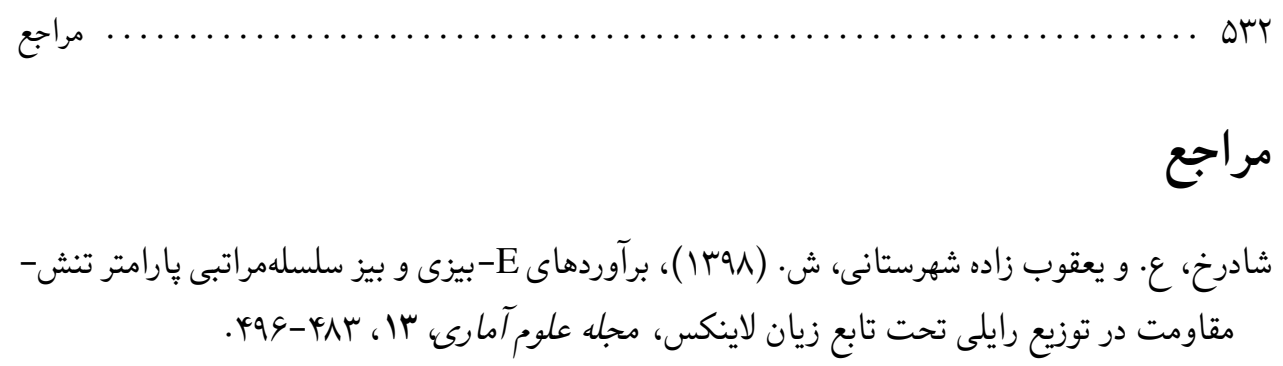

Ahmadi, K. and Ghafouri, S. (2019), Reliability Estimation in a Multicomponent Stress-Strength Model under Generalized Half-Normal Distribution Based on Progressive type-II Censoring, Journal of Statistical Computation and Simulation, 89, 2505-2548.

Asgharzadeh, A., Valiollahi, R. and Raqab, M. Z. (2011), Stress-Strength Reliability of Weibull Distribution Based on Progressively Censored Samples, SORT, 35, 103-124.

Balakrishnan, N. and Aggarwala, R. (2000), Progressive Censoring: Theory, Methods and Applications, Boston, MA, USA: Birkhauser.

Chen, M. H. and Shao, Q. M. (1999), Monte Carlo Estimation of Bayesian Credible and HPD Intervals, Journal of Computational and Graphical Statistics, 8, 69-92.

Efron, B. (1988), Logistic Regression, Survival Analysis, and the KaplanMeier Curve, Journal of the American Statistical Association, 83, 414-425

Jha, M. K., Dey, S., Alotaibi, R. M. and Tripathi, Y. M. (2020), Reliability Estimation of a Multicomponent Stress-Strength Model for Unit Gompertz Distribution under Progressive Type II Censoring, Quality and Reliability Engineering International, DOI: 10.1002/qre.2610.

Kizilaslan, F. and Nadar, M. (2018), Estimation of Reliability in a Mul- 
ticomponent Stress-Strength Model Based on a Bivariate Kumaraswamy Distribution, Statistical Papers, 59, 307-340.

Kohansal, A. (2020), Bayesian and Classical Estimation of $R=P(Y<X)$ Based on Burr Type XII Distribution under Hybrid Progressive Censored Samples, Communications in Statistics-Theory and Methods, 49, 10431081.

Kohansal, A. and Rezakhah, S. (2019), Inference of $R=P(Y<X)$ For Two-Parameter Rayleigh Distribution Based on Progressively Censored Samples, Statistics, 53, 81-100.

Kohansal, A. (2019), On Estimation of Reliability in a Multicomponent Stress-Strength Model for a Kumaraswamy Distribution Based on Progressively Censored Sample, Statistical Papers, 60, 2185-2224.

Kundu, D. and Joarder, A. (2006), Analysis of Type-II Progressively Hybrid Censored Data, Computational Statistics and Data Analysis, 50, 25092528.

Lindley, D. V. (1980), Approximate Bayesian Methods, Trabajos de Estadistica, 3, 281-288.

Mahmoud, M. A. W., El-Sagheer, R. M., Soliman, A. A. and Abd Ellah, A. H. (2016), Bayesian Estimation of $P[Y<X]$ Based on Record Values From the Lomax Distribution and MCMC Technique, Journal of Modern Applied Statistical Methods, 15, 488-510.

Maurya, R. K. and Tripathi, Y. M. (2019) Reliability Estimation in a Multicomponent Stress-Strength Model for Burr XII Distribution under Progressive Censoring, Brazilian Journal of Probability and Statistics, Accepted. 
Shoaee, Sh. and Khorram, E. (2016), Statistical Inference of $R=P(Y<X)$ For Weibull Distribution under Type-II Progressively Hybrid Censored Data, Journal of Statistical Computation and Simulation, 86, 3815-3834.

Shoaee, Sh. and Khorram, E. (2015), Stress-Strength Reliability of a TwoParameter Bathtub-Shaped Lifetime Distribution Based on Progressively Censored Samples, Communications in Statistics-Theory and Methods, 44, 5306-5328.

Yadav, A. S., Singh, S. K. and Singh, U. (2019), Bayesian Estimation of Stress-Strength Reliability for Lomax Distribution under Type-II Hybrid Censored Data Using Asymmetric Loss Function, Life Cycle Reliability and Safety Engineering, 8, 257-267. 
Journal of Statistical Sciences, Autumn and Winter, 2020

Vol. 14, No. 2, pp 505-534

DOI: $10.29252 /$ jss.13.2.505

\title{
Bayesian Estimation of Stress-Strength Parameter under Progressive Hybrid Censored Sample in Lomax Distribu- tion
}

\author{
Kohansal، A. ${ }^{1}$ ، Alemohammad، N. ${ }^{2}$, Azizzadeh، F. ${ }^{3}$ \\ ${ }^{1}$ Department of Statistics, Imam Khomeini International University, Qazvin, \\ Iran. \\ ${ }^{2}$ Department of Mathematics and Computer Sciences, Shahed University, \\ Tehran, Iran. \\ ${ }^{3}$ Department of Financial Mathematics, Kharazmi University, Teheran, Iran.
}

\begin{abstract}
The Bayesian estimation of the stress-strength parameter in Lomax distribution under the progressive hybrid censored sample is considered in three cases. First, assuming the stress and strength are two random variables with a common scale and different shape parameters. The Bayesian estimations of these parameters are approximated by Lindley method and the Gibbs algorithm. Second, assuming the scale parameter is known, the exact Bayes estimation of the stress-strength parameter is obtained. Third, assuming all parameters are unknown, the Bayesian estimation of the stressstrength parameter is derived via the Gibbs algorithm. Also, the maximum likelihood estimations are calculated, and the usefulness of the Bayesian estimations is confirmed, in comparison with them. Finally, the different methods are evaluated utilizing the Monte Carlo simulation and one real data set is analyzed.
\end{abstract}

Keywords: Stress-strength parameter, Lindley's approximation, Lomax distribution, Progressive hybrid censored.

Mathematics Subject Classification (2010): 62N01, 62N05, 62F15 